\title{
Evaluación de la interfaz ciencia-política a nivel municipal en la implementación de la Agenda Local $2 \mathrm{I}$ en Michoacán (México)
}

\author{
Evaluation of the Science-Policy Interface at the Local Level in the Implementation of Local \\ Agenda 21 in Michoacan (Mexico)
}

\author{
Ana L. Burgos y Gerardo Bocco*
}

\begin{abstract}
Resumen: Este trabajo evaluó la interfaz ciencia-política (ICP) propiciada por el instrumento Agenda Local 21 en su versión mexicana (Agenda Desde lo Local); que conjuntó a funcionarios estatales (FE), municipales (FM) y expertos académicos (EX) sobre la gestión para el desarrollo sostenible en los municipios de Michoacán (México) entre 2008 y 2013. La evaluación utilizó un marco conceptual con cuatro categorías analíticas integradas por 16 factores y 39 atributos. En la categoría de factores sistémicos, los objetivos y procesos de la ICP mostraron fortalezas, pero las salidas fueron débiles. En la categoría de factores actitudinales, la distancia cognitiva y niveles de realidad entre FM Y EX estuvieron diferenciados. La motivación, el compromiso y la percepción del otro fueron excelentes en FE, pero en FM y EX se expresaron insuficientemente. La evaluación identificó lecciones aprendidas de interés en el diseño de instrumentos de política pública para el encuentro entre la ciencia y la política.

Palabras clave: interfaz ciencia-política, sistemas de conocimiento, municipalismo, gobierno local, diseño de políticas, gobernanza, desarrollo sostenible, transdisciplina.
\end{abstract}

*Ana L. Burgos es investigadora titular "A" del Centro de Investigaciones en Geografía Ambiental (CIGA) de la Universidad Nacional Autónoma de México (unAm) Campus Morelia, Antigua Carretera a Pátzcuaro 8701, colonia Ex-Hacienda de San José de la Huerta, 58190, Morelia, Michoacán. Correo-e: aburgos@ciga.unam.mx. Tel: +52 443322 3833. oRcID: 0000-0002-0666-4161. Gerardo Bocco es investigador titular "C" en el mismo centro de investigación (CIGA-UnAM Campus Morelia). Correo-e: gbocco@ ciga.unam.mx. Tel: +52 443 3223834. ORCID: 0000-0003-4542-3544. Los autores agradecen a Rosaura Paez Bistrain y Tamara Ortiz Ávila, quienes integraron el equipo de expertos académicos de la UNAM de manera sostenida durante los seis ańos de trabajo, y propiciaron momentos enriquecedores de análisis e intercambio de opiniones. A Gabriela Ruiz López, coordinadora del Programa Agenda Desde lo Local en el Centro para el Desarrollo Municipal (Cedemun) del estado de Michoacán en 2008-2012, y a Eliacim Cańada Rangel y Mauricio Mata de 2012 a 2013, por su apertura y facilitación de datos confiables sobre el proceso. Las sugerencias de dos revisores anónimos mejoraron sustancialmente la claridad del artículo.

Artículo recibido el 1 de marzo de 2019 y aceptado para su publicación el 28 de febrero de 2020.

DoI: http://dx.doi.org/10.29265/gypp.v30i1.819 
Abstract: This work evaluated the Science-Policy Interface (sPI) promoted by the political instrument Agenda Local 21 in its Mexican version (called Agenda Desde lo Local) which articulated state officials (so), municipal officials (MO), and academic experts (Ex) on local management for sustainable development and were implemented in Michoacan (Mexico) between 2008 and 2013. The evaluation was conducted through a conceptual framework with four analytical categories spanning 16 factors and 39 attributes. Among the systemic factors, the objectives and processes of the interface showed strength, but the outputs had uncertain impacts. The attitudinal factors of involved actors showed high cognitive distance and very differentiated levels of reality between MO and Ex. The motivation, commitment, and perception of the others were excellent in so, but the remaining actors showed weak performance. The evaluation led to lessons learned of interest in the design of political instruments that promote the links between science and policy.

Keywords: science-policy interface; knowledge systems, municipalism, local government, policy design, governance, sustainable development, transdiscipline.

\section{INTRODUCCIÓN}

- $\mathrm{n}$ la sociedad contemporánea, el diseño, la implementación y el seguimiento de políticas públicas enfrentan altos niveles de complejidad e incertidumbre. Por ello, el diálogo entre la ciencia y la política se ha reconocido como un requisito urgente para mejorar la toma de decisiones con base en evidencias confiables y en marcos heurísticos novedosos (Funtowics y Ravetz, 1993; Engels, 2005; Strydom et al., 2010). A pesar de ello, la relación entre la ciencia y la política continúa mostrando dificultades para alcanzar una relación mutuamente fructífera (Nursey-Bray et al., 2014; Gluckman, 2016; Von der Heyden et al., 2016; More, 2019).

La interfaz ciencia-política (ICP) es un objeto de estudio de creciente interés, definido como el proceso de interacción directa entre científicos y expertos entrenados científicamente, por un lado, y agentes de organizaciones que formulan e implementan políticas públicas, por el otro (Broström y Mckelvey, 2018). Se trata de un proceso social que crea sistemas de conocimiento de frontera para el intercambio, coevolución y construcción conjunta de conocimientos, con el objetivo de enriquecer la toma de decisiones (Cash et al., 2002; Van den Hove, 2007). Actualmente, se trata de un campo de investigación de creciente dinamismo.

En los últimos años, los estudios sobre la ICP han cobrado mayor importancia en el ámbito de las ciencias ambientales y de la sostenibilidad. La creciente crisis ambiental y la necesaria gobernanza para reducir los impactos negativos sobre el planeta dinamizaron la creación de interfaces y organizaciones de frontera cuyo propósito es promover políticas públicas capaces de orientar transiciones hacia 
futuros más sostenibles (White, Corley y White, 2008; Strydom et al., 2010; Sarkki et al., 2015).Tales son los casos del Panel Intergubernamental sobre el Cambio Climático (IPCC, por sus siglas en inglés) instalado en 1988 (Tonn, 2007); y la Plataforma Intergubernamental Científico-Normativa sobre Diversidad Biológica y Servicios de los Ecosistemas conocida como IPBES, creada en 2010 (Perrings et al., 2011; Vohland et al., 2011). Más recientemente, en países industrializados y periféricos, se han implementado ICPs para atender problemáticas ambientales a nivel nacional (Huitema y Turnhout, 2009; Swilling, 2014; Scarano et al., 2019). Los estudios sobre las ICPs en diferentes experiencias han indagado en los factores que intervienen en su construcción y efectividad, con el propósito de contribuir al mejor diseño de políticas públicas e instrumentos para fomentar el encuentro entre la ciencia y la política.

En contraste con los niveles internacional y nacional, las políticas públicas para crear ICPs a nivel local han sido más escasas. Una iniciativa fue la denominada Agenda Local 21 (AL21) — un instrumento derivado del capítulo 28 de la Agenda 21 aprobada en la Cumbre de la Tierra de Río en 1992- para promover el desarrollo sostenible desde la acción de los gobiernos locales (Lafferty y Eckerberg, 1998; García-Añón, 2004). Las AL21 se implementaron principalmente en países europeos, donde se promovió la participación de las sociedades locales (Selman y Parker, 1997; Sancassiani, 2005; Coenen, 2009), y se incluyó al sector científico-académico (Kitchen, Whitney y Littlewood, 1997). Estas iniciativas detonaron la creación a nivel local de ICPs novedosas.

En México, la AL21 se materializó en el instrumento denominado Programa Agenda Desde lo Local (ADLL) lanzado en 2004. La ADLL se implementó en 29 entidades federativas, entre ellas el estado de Michoacán, donde operó entre 2008 y 2013. Michoacán, al igual que otros estados mexicanos, muestra una marcada debilidad institucional a nivel estatal, y escasas capacidades técnicas y de gestión en los gobiernos municipales. De modo que el instrumento de la ADLL se presentó como una oportunidad para mejorar dicha condición.

Desde un análisis retrospectivo, el propósito de este trabajo es evaluar la interfaz ciencia-política (ICP) promovida en la implementación del Programa "Agenda Desde lo Local" (ADLL) en el estado de Michoacán (México) en el periodo 2008-2013. Este trabajo aporta tres contribuciones principales. Primero, presenta un marco de evaluación integrador de la ICP, que sintetiza los factores reconocidos en la literatura como determinantes para elevar su efectividad. El marco de evaluación da rigor al objeto de estudio, y constituye una contribución metodológica para aquellos interesados en estudios de gobernanza. Además, el 
trabajo documenta una experiencia de una ICP a nivel de los gobiernos municipales en México, siendo uno de los escasos trabajos empíricos disponibles hasta el momento. Por último, la evaluación del caso identifica las limitaciones y lecciones aprendidas en la construcción de la ICP a nivel local en el contexto mexicano. Como toda evaluación, este trabajo contribuye a mejorar el diseño e implementación de instrumentos de política pública. En particular, es útil para aquellos instrumentos que pudieran fortalecer la gestión de los gobiernos municipales con los recursos intelectuales y cognitivos de las universidades e instituciones de educación superior de arraigo regional. La construcción de ICPs más efectivas a nivel local tiene relevancia ante los retos de los Objetivos para el Desarrollo Sostenible expresados en la denominada Agenda 2030, que demandan un papel protagónico de los gobiernos municipales (Slack, 2015; Fenton y Gustafsson, 2017; Ojeda-Medina, 2020).

\section{ANTECEDENTES: LA INTERFAZ CIENCIA-POLÍTICA}

El interés en la relación entre la ciencia y la política se instaló a principios de los años noventa con la aproximación denominada Ciencia Post-Normal, propuesta por Silvio Funtowics y Jerzy Ravetz (1993). Ante la alta complejidad del mundo contemporáneo, estos autores alertaron sobre la necesidad de moverse desde una ciencia aplicada o de consultoría, a una ciencia capaz de comprender y atender las necesidades de los tomadores de decisiones, bajo altos niveles de incertidumbre y urgencia, intereses en juego y valores en conflicto. Con ello, la Ciencia Post-Normal establecía la interfaz ciencia-política (ICP) como un objeto de estudio que actualmente es central en los conceptos de gobernanza colaborativa (Koontz, 2019) y de gobernanza ambiental (Sarkki et al., 2019). La construcción de ICPs interviene en otros campos de interés para la gestión y la política pública, como el diseño de instrumentos políticos para la creación de sistemas de innovación (Lundvall et al., 2002). Los sistemas y ecosistemas de innovación emergen de interacciones efectivas entre universidades, gobierno e industria, tal como lo expresa el modelo de la triple hélice (Leydesdorff y Etzkowitz,1998; Etzkowitz y Leydesdorff, 2000). Para acotar este trabajo, el abordaje de la ICP en el campo de la teoría de la innovación queda fuera de los alcances de este texto.

En los últimos veinte años, el interés sobre la ICP creció de manera notable en las ciencias ambientales, la gestión ambiental y el desarrollo sostenible. Con un llamativo acercamiento hacia las ciencias políticas, estos campos de investigación se han enfocado en identificar debilidades y desafíos de los sistemas de conocimiento de frontera, con el propósito de facilitar el encuentro para la coproducción 
de conocimientos entre científicos tanto ambientales como sociales, y decisores (Nesshöver et al., 2013; Saviano et al., 2019). Del lado de las ciencias políticas, administrativas o afines, en cambio, el interés sobre la ICP se ha enfocado principalmente en las características que debe reunir el conocimiento científico para ser útil a las políticas públicas (Cash et al., 2002; Heink et al., 2015), así como en el uso del conocimiento científico en la toma de decisiones y en la percepción de los usuarios (Koontz, 2019; Ramírez y Belcher, 2019; Zheng et al., 2019). El creciente interés sobre la ICP produjo en los últimos años una gran cantidad de desarrollos conceptuales de profundidad y evidencias emergentes de casos de estudio en contextos específicos de gobernanza.

\section{MARCO DE EVALUACIÓN DE LA ICP}

Para abordar la evaluación de la ICP con un enfoque integrador, se estableció un marco conceptual que incluyó cuatro categorías analíticas (diagrama 1): a) los componentes sistémicos de la interfaz; $b$ ) las actitudes de los involucrados; $c$ ) las características del conocimiento, y d) los dilemas. En estas categorías se consideraron 16 factores que son reconocidos en la literatura especializada como determinantes de la efectividad de la interfaz. Para hacer operativa la evaluación, cada uno de los 16 factores fue asociado con uno o más atributos específicos. Los atributos se definieron como hechos observables, medibles e interpretables mediante datos de tipo cuantitativo, cualitativo o categórico. A continuación, se revisan las bases conceptuales y teóricas de las cuatro categorías analíticas consideradas, así como los antecedentes conceptuales de los factores y sus atributos respectivos.

\section{Componentes sistémicos de la interfaz}

En esta categoría se incluyeron cuatro factores derivados de una conceptualización sistémica de la ICP (Sarkki et al., 2015): el objetivo, la estructura, los procesos y las salidas (diagrama 1). Los factores sistémicos permiten reconocer los alcances de la interfaz, así como las barreras para su construcción y sostenimiento.

El objetivo es un factor sistémico que ha sido finamente conceptualizado en la Teoría de Objetos de Frontera (diagrama 1). El objeto de frontera es definido como un objeto científico abstracto o concreto (idea, concepto, noción, portadores de información, entre otros), que se constituye como una representación simbólica instalada en la intersección entre mundos sociales diferenciados, que satisface los requerimientos de información de todos ellos (Star y Griesemer, 1989). El objeto de frontera es considerado el núcleo de la construcción de interfaces entre comunidades de práctica diferenciadas, porque es un punto de 


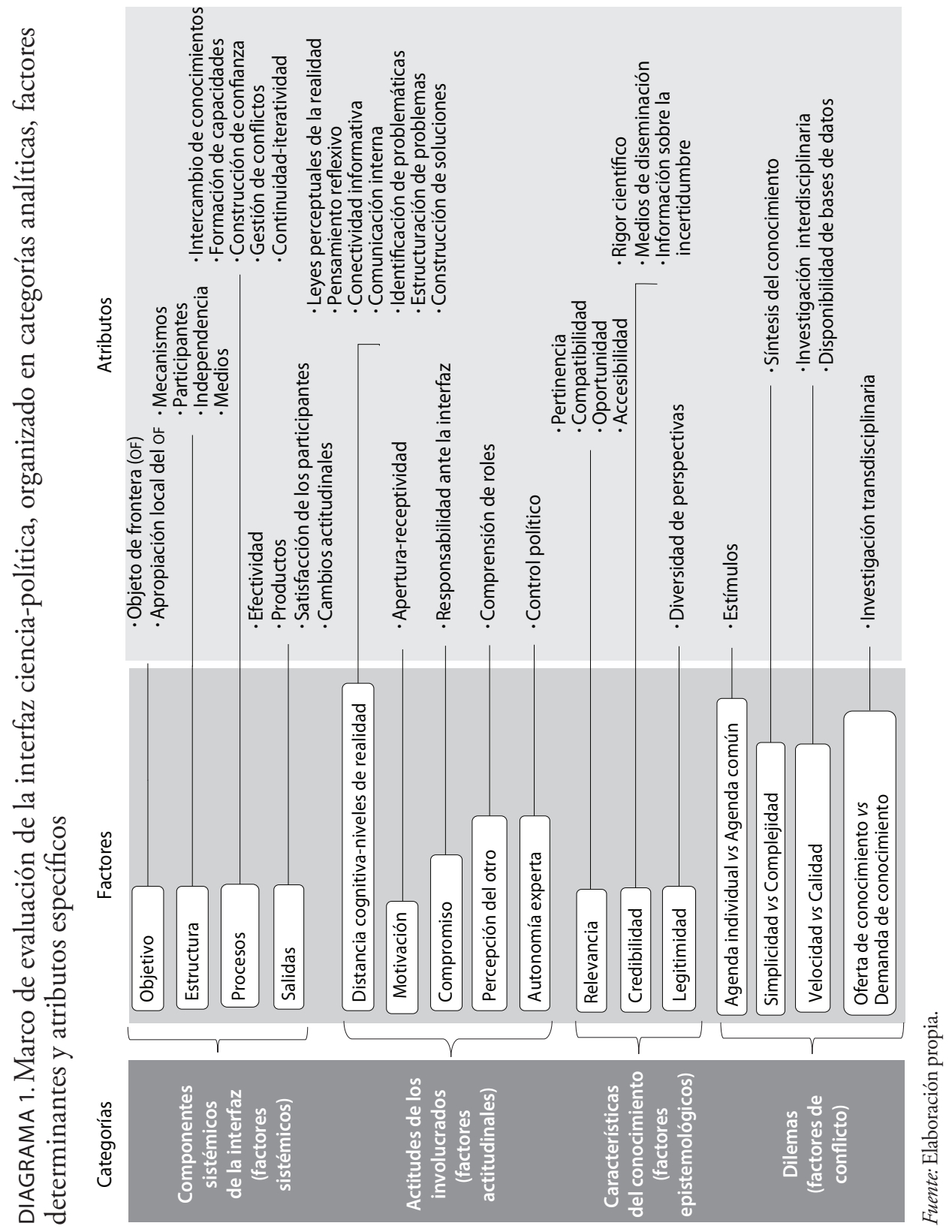


referencia concreto en el que los involucrados encuentran un interés mutuo, el cual está más allá de la obligación de lograr un consenso entre las partes (Star, 2010). Por ejemplo, Steger y colaboradores (2018) discutieron a profundidad el alcance del constructo conceptual "servicios ecosistémicos" como un objeto de frontera. Estos investigadores propusieron que el objeto de frontera no es "algo rígido", sino un proceso con diversas fases que abarca desde la formulación inicial del objeto de frontera como una representación abstracta y flexible que es compartida entre diferentes comunidades, hasta su normalización mediante una infraestructura conceptual que pierde flexibilidad a través de categorías, pero que facilita el diálogo entre sectores. Dada la importancia del objeto de frontera en la ICP, el marco de evaluación incluye dos atributos relacionados con este: la formulación inicial del objeto de frontera, y el nivel de apropiación por los involucrados (diagrama 1).

La estructura es el factor sistémico que determina las formas y los medios para la construcción y el sostenimiento de la interfaz. Sarkki y colaboradores (2015) propusieron algunos atributos relevantes que fueron incluidos en este marco de evaluación (diagrama 1). El primero se refiere a los mecanismos institucionales (normas, reglas, procedimientos) que articulan la creación y el funcionamiento de la interfaz. El segundo atributo observa los participantes involucrados (e.g. los actores a ambos lados de la interfaz), dado que la presencia o ausencia de los sujetos que efectivamente deben involucrarse constituye una barrera potencial a su efectividad. El tercer atributo es el grado de independencia de la estructura, definido como la libertad y ausencia de control externo, así como el grado de neutralidad y transparencia sobre posibles sesgos (Sarkki et al., 2015). Un último atributo estructural son los medios disponibles para su funcionamiento, tanto tangibles (e.g. financiamiento, espacios físicos) como intangibles (e.g. tiempo). Los cuatro atributos estructurales revisten gran importancia porque crean las condiciones concretas para la implementación de la ICP.

El factor sistémico de procesos aborda las acciones e interacciones que producen las salidas de la interfaz (Sarkki et al., 2015). Es un componente esencial por lo que en este marco de evaluación se le desarrolla mediante cinco atributos funcionales (diagrama 1). El primer atributo es el intercambio e integración de conocimientos, un proceso fundamental en la investigación transdisciplinaria (Scholz y Steiner, 2015). El modelo lineal en el que la ciencia transfiere de manera unilateral el conocimiento ha quedado sustituido por aquel que reconoce la importancia de los flujos multidireccionales que emergen cuando la interfaz es concebida como una conversación (Young et al., 2014). El segundo atributo 
funcional es la formación de capacidades para romper con el pensamiento compartimentado (en inglés, silo thinking), y entender a la ICP como un ambiente de diálogo, aprendizaje mutuo y colaboración (Tinch et al., 2018). Otros dos atributos funcionales son la construcción de confianza y la gestión de conflictos (diagrama 1); ambos son de gran relevancia en contextos de debilidad institucional. Finalmente, el quinto atributo funcional observa la continuidad-iteratividad de la interfaz. La continuidad se pondera mediante la participación de las mismas personas y la repetición de procesos internos a largo plazo (Tinch et al., 2018), mientras que la iteratividad indica el modo en que la interfaz evoluciona (Sarkki et al., 2015). Los cinco atributos funcionales son interdependientes, aunque el atributo de continuidad-iteratividad es subyacente a la ocurrencia de los demás.

El factor de salidas de la interfaz es el último componente sistémico de los considerados en el marco de evaluación (diagrama 1). En el enfoque sistémico, las salidas corresponden con productos específicos que resultan de los procesos, así como cambios ocurridos en los componentes del sistema y en su entorno. En una ICP, las salidas pueden evaluarse por los efectos e impactos sobre el objeto de frontera y también por los efectos directos sobre las partes en interacción. Sarkki y colaboradores (2015) propusieron que las salidas incluyen reportes, bases de datos, recomendaciones, entre otros portadores de información que pueden ser útiles a los tomadores de decisiones. En este marco de evaluación se consideraron cuatro atributos de salida (diagrama 1). El primero es la efectividad, que reconoce el alcance que la interfaz ha tenido sobre el objeto de frontera. El segundo atributo de las salidas es la calidad y cantidad de productos de conocimiento generados. Los últimos dos atributos se enfocan en la satisfacción de los participantes, la cual emana de las percepciones de los involucrados y de los cambios ocurridos en las actitudes de los participantes hacia la interfaz. Estos últimos atributos están muy relacionados con la siguiente categoría de análisis.

\section{Actitudes de los involucrados}

Esta categoría analítica se refiere a los aspectos cognitivos y actitudinales de los expertos científicos y los políticos involucrados en la interfaz, los cuales están relacionados con la denominada actitud individual transdisciplinaria (Nicolescu, 2002; Augsburg, 2014). En el marco de evaluación se consideraron cinco factores actitudinales, que son aprehensibles mediante 11 atributos (diagrama 1). Cabe señalar que en esta categoría, los atributos son de difícil valoración por su naturaleza abstracta y fuertemente subjetiva. 
Un primer factor actitudinal es la distancia cognitiva-niveles de realidad en los cuales se sitúan los actores implicados. El concepto de distancia cognitiva fue propuesto por Broström y McKelvey (2018) para designar al grado de separación entre dos organizaciones o individuos, en términos de sus bases de conocimiento, valores, normas y marcos heurísticos. Por su parte, el nivel de realidad es un concepto desarrollado en la epistemología de la transdisciplinariedad de Basarab Nicolescu $(2002,2014)$, y señala la forma en la que los individuos construyen modelos mentales sobre el mundo fenoménico, los cuales responden a patrones cognitivos que son determinantes de la percepción del sujeto. Esto implica que dos niveles de realidad son diferentes si al pasar de uno al otro hay una ruptura en los conceptos fundamentales que los constituyen y en las leyes que los gobiernan (Nicolescu, 2012). De acuerdo con ello, diferentes sectores sociales podrían ubicarse en niveles de realidad diferenciados; y con ello construir formas de comprensión pragmática también diferenciadas que inciden en la delimitación de problemas y en la construcción de soluciones. De acuerdo con Max-Neef (2005), los flujos de información que resultan del diálogo entre sectores operan como flujos de conciencia entre estos diferentes niveles de realidad. Con base en estas premisas conceptuales, la evaluación del factor de distancia cognitiva-niveles de realidad se basó en siete atributos (diagrama 1). La revisión detallada de los antecedentes conceptuales de cada atributo excede las posibilidades de este texto.

Un segundo factor actitudinal es la motivación de los actores hacia la interfaz, que puede ponderarse mediante el atributo de apertura-receptividad (diagrama 1). Este atributo depende tanto de estímulos institucionales a ambos lados de la interfaz, como de convicciones personales de los participantes. A pesar de la demostrada relevancia de la ICP, la apertura de investigadores y académicos hacia esta interacción dista mucho de ser la requerida (Young et al., 2014). En los decisores, la receptividad hacia el sector académico depende en gran medida de la cercanía del objeto de frontera con sus agendas e intereses políticos, y de la forma en que los funcionarios valoran a la actividad y conocimiento científicos (Jones, Fischhoff y Lach, 1999).

El tercer factor actitudinal es el compromiso de los actores hacia la interfaz, que en el marco de evaluación se refleja en el atributo de responsabilidad (diagrama 1). Este atributo se puede ponderar con el nivel de respuesta observado hacia lo acordado entre las partes y hacia las necesidades para la construcción de la interfaz.

El cuarto factor actitudinal es la percepción del otro, que se pondera con el atributo de comprensión de roles. Los científicos deben incrementar su compren- 
sión de cómo se navega la toma de decisiones, y los funcionarios necesitan comprender los procesos de producción de conocimiento científico. Este atributo se fomenta mediante el diálogo y la capacitación de las partes.

Finalmente, un quinto factor actitudinal es la autonomía experta. Con ese término Broström y McKelvey (2018) designaron al grado de control formal o informal que ejercen los hacedores de políticas sobre las actividades de los expertos. El nivel de autonomía experta varía desde muy bajo cuando el sector gubernamental tiene un control cerrado de la actividad científica, hasta un nivel muy alto cuando existe una separación absoluta de la actividad científica y los intereses del sector gubernamental.

\section{Características del conocimiento}

El fin último de la ICP es propiciar el uso de conocimiento científico para la toma de decisiones y el mejor diseño de políticas públicas e instrumentos. En este sentido, se han reconocido tres propiedades que influyen en el logro de dicho propósito (diagrama 1): la relevancia, la credibilidad y la legitimidad del conocimiento (Cash et al., 2003). Los tres factores son interdependientes y también son afectados por los factores de conflicto (dilemas) presentados en la siguiente sección. Dada su importancia, estos tres factores son recurrentemente analizados en la literatura (Heink et al., 2015; Sarkki et al., 2014; Sarkki et al., 2015).

El factor de relevancia es altamente dependiente del contexto de la interfaz, y puede reflejarse mediante tres atributos (diagrama 1). El primero es la pertinencia del conocimiento, que se refleja en la proximidad del conocimiento con las agendas políticas, y la forma en que el objeto de frontera está instalado y apropiado por los decisores. El segundo atributo es la compatibilidad de las escalas entre aquella del conocimiento producido y aquella del nivel de organización (órdenes de gobierno) en el que opera la interfaz. El logro de una adecuada compatibilidad de escalas requiere mayores capacidades en el sector académico para integrar y desagregar información de acuerdo con el nivel de operación del sector político involucrado en la interfaz. El tercer atributo es la oportunidad o timing, es decir, el momento en el que el conocimiento es hecho disponible en relación con los tiempos políticos. El cuarto atributo es la accesibilidad del conocimiento desde el sector gubernamental (Jones et al., 1999; Cash et al., 2002).

$\mathrm{El}$ factor de credibilidad del conocimiento depende de las propiedades de la información, en términos de la calidad de datos, validez de modelos y consistencia, así como de la forma en la cual la información es presentada (Heink et al., 2015). Dichas propiedades pueden ser ponderadas mediante, al menos, tres atri- 
butos (diagrama 1): el rigor científico, los medios de diseminación y la información sobre la incertidumbre. Estos atributos demandan gran atención y cuidado desde el sector científico (More, 2019).

Finalmente, el factor de legitimidad está relacionado con la cercanía a principios preestablecidos (Heink et al., 2015), así como con la representatividad, en términos de las voces y perspectivas consideradas en el proceso de producción del conocimiento (Cash et al., 2002).

\section{Dilemas}

La construcción de la ICP es afectada por dilemas o factores de conflicto, que fueron analizados a detalle por Sarkki y colaboradores (2014), y que por su relevancia deben ser incluidos en un marco de evaluación (diagrama 1).

Un primer dilema es la contradicción entre la agenda personal y la agenda común. Desde el sector científico, innumerables artículos han discutido las trabas para el involucramiento y compromiso de los científicos en interfaces con la sociedad. Trabas similares se presentan en el sector gubernamental, en el que las agendas sectoriales por un lado, y los sesgos en la visión institucional o personal de los funcionarios, por el otro, inhiben la asignación de tiempo y apertura a la interacción con expertos. Más allá de los factores actitudinales antes revisados, este dilema puede reducirse mediante estímulos o incentivos institucionales como el reconocimiento público de la colaboración, y la inclusión de la participación en ICPs como criterio de evaluación académica y de la función pública.

Otros dos factores de conflicto son la relación entre simplificación y complejidad del conocimiento, y entre velocidad y calidad de la información (diagrama 1). Ambos dilemas están mutuamente relacionados, y su resolución requiere cambios profundos en las prácticas científicas establecidas. Los atributos que permiten ponderarlos son, por ejemplo, la síntesis del conocimiento disponible para reducir la complejidad sin caer en la simplificación; la disponibilidad de bases de datos organizadas y actualizadas, y el funcionamiento de grupos interdisciplinarios con alta capacidad intelectual y entrenamiento para la producción rápida de conocimiento pertinente.

El último dilema es el conocimiento por oferta (push) y por demanda (pull). Mientras el primero cuenta con mayor credibilidad porque es generado bajo los tiempos científicos, el segundo cuenta con mayor relevancia pues es solicitado para atender problemas definidos por los decisores (Sarkki et al., 2014). Este dilema se resuelve por la implementación de grupos de investigación transdisciplinaria que propicien la formulación conjunta de un problema concreto de interés y la 
colaboración ágil para la coproducción de conocimientos pertinentes (Scholz y Steiner, 2015; Fam, Neuhauser y Gibbs, 2018; Tinch et al., 2018).

\section{ANTECEDENTES: LA AGENDA LOCAL 21 EN MÉXICO}

México se sumó a los esfuerzos internacionales para impulsar la Agenda Local 21 (AL21) en el ańo 2004, bajo la presidencia de Vicente Fox. La iniciativa fue desarrollada por el Instituto Nacional para el Federalismo (Inafed), que es un organismo desconcentrado del gobierno federal (Inafed, 2004; Pińa, 2013), el cual diseńó el instrumento denominado Programa Agenda Desde lo Local o ADLL con respaldo de estudiosos del municipalismo en México (e.g. Ziccardi y Saltalamacchia, 1997; Merino, 2005). El programa fue implementado por los Centros para el Desarrollo Municipal (Cedemun) o equivalentes de cada entidad federativa, y coordinado por un órgano denominado Consejo Nacional Desde Lo Local, integrado por autoridades federales, enlaces estatales y representantes de las Instituciones de Educación Superior (IES) por regiones del país (Inafed, 2012). En 2014, bajo la presidencia de Enrique Peña Nieto (2012-2018), el Inafed reformuló la ADLL y la transformó en el Programa Agenda para el Desarrollo Municipal. Este instrumento ya no estuvo orientado a fomentar el desarrollo local sostenible, sino más bien a mejorar la prestación de servicios públicos por el municipio, una obligación mandatada en el artículo 115 de la Constitución mexicana (Inafed, 2014).

\section{Mecánica y aplicación de la ADLL}

La ADLL fue lanzada como "un programa y una metodología que parte de un diagnóstico integral de la situación en la que se encuentran los municipios en materia de desarrollo local, para orientar, con base en este, acciones de los tres órdenes de gobierno que mejoren el nivel y la calidad de vida de la población" (Inafed, 2004). La mecánica de implementación constaba de cuatro etapas: 1) autodiagnóstico; 2) verificación; 3) atención de áreas de oportunidad, y 4) expedición de certificados. El autodiagnóstico era realizado por los propios funcionarios municipales en torno a cuatro dimensiones del desarrollo sostenible: la dimensión institucional, la económica, la social y la ambiental. Estas dimensiones eran valoradas mediante 39 indicadores y 270 parámetros que se referían a los procedimientos y acciones en la gestión local para atender los temas de la agenda. Los funcionarios locales debían revisar a conciencia cada parámetro y formular un autodiagnóstico de la gestión durante el último año. El procedimiento utilizaba un código de colores: el verde indicaba un cumplimiento total de los requisitos del parámetro, el amarillo señalaba el cumplimiento parcial y el rojo indicaba la ausencia de acciones. 
El ayuntamiento debía organizar la evidencia documental (datos, informes, comprobantes) que demostraba las acciones realizadas. La segunda etapa de verificación consistía en la validación del autodiagnóstico por un órgano independiente y neutral. Aquí entraban en juego las IEs públicas y privadas con arraigo en cada estado, quienes revisaban minuciosamente la evidencia documental en la que el municipio basaba su autodiagnóstico, para emitir un dictamen y, en su caso, recomendaciones. En la tercera etapa, el ayuntamiento seleccionaba áreas de mejora para atender en el corto plazo. Los resultados de la verificación eran enviados al Consejo Nacional Desde lo Local, el cual expedía los certificados verdes. Una vez al año, se realizaba un foro nacional en el que se entregaban dichos certificados a los presidentes municipales, y se nombraban los ayuntamientos merecedores del Premio al Buen Gobierno Municipal, cuando un ayuntamiento había cumplimentado los 39 indicadores. En estos foros también se ofrecían ponencias, estudios de caso exitosos y otras actividades para el fortalecimiento del municipalismo.

A partir del diseño del instrumento por el Inafed (nivel federal), el Programa ADLL se materializó con la interacción directa de tres actores principales: el gobierno estatal de cada entidad federativa como responsable de su implementación, los gobiernos municipales y las IEs del estado. La etapa de verificación creaba las condiciones para la interacción directa de funcionarios de nivel municipal, con expertos científicos y académicos. En 2012, la ADLL llegó a funcionar en 29 entidades federativas, con la participación de 105 IEs (Inafed, 2012). La instrumentación de la ICP para la ADLL, si bien normada en los lineamientos generales, se manifestó con particularidades y matices en cada entidad federativa.

\section{EL PROGRAMA ADLL EN MICHOACÁN}

El estado de Michoacán de Ocampo tiene una extensión de $58599 \mathrm{~km}^{2}$, equivalente a 2.9 por ciento de la superficie de México; y está conformado por 113 municipios integrados en diez regiones administrativas. En 2015 contaba con una población de 4.5 millones de habitantes (3.8 por ciento del total nacional), que lo ubicaba en el noveno puesto por su tamaño poblacional (INEGI, 2016).

Michoacán se incorporó al Programa ADLL en el año 2008 durante el gobierno de Leonel Godoy Rangel ${ }^{1}$ (periodo 2008-2012), quien encomendó la implementación al Centro para el Desarrollo Municipal (Cedemun-Mich). ${ }^{2}$ La ADLl se

\footnotetext{
${ }^{1}$ Perteneciente al Partido de la Revolución Democrática (PRD).

${ }^{2}$ Durante el periodo 2008-2012, el Cedemun-Michoacán estuvo a cargo de Mario Ensástiga Santiago, quien delegó la implementación de la ADLL en Gabriela Ruiz López.
} 
GRÁFICA 1. Posición nacional de los estados de la Región Centro-Occidente y de Guerrero por nivel decreciente de marginación entre 1990 y 2015

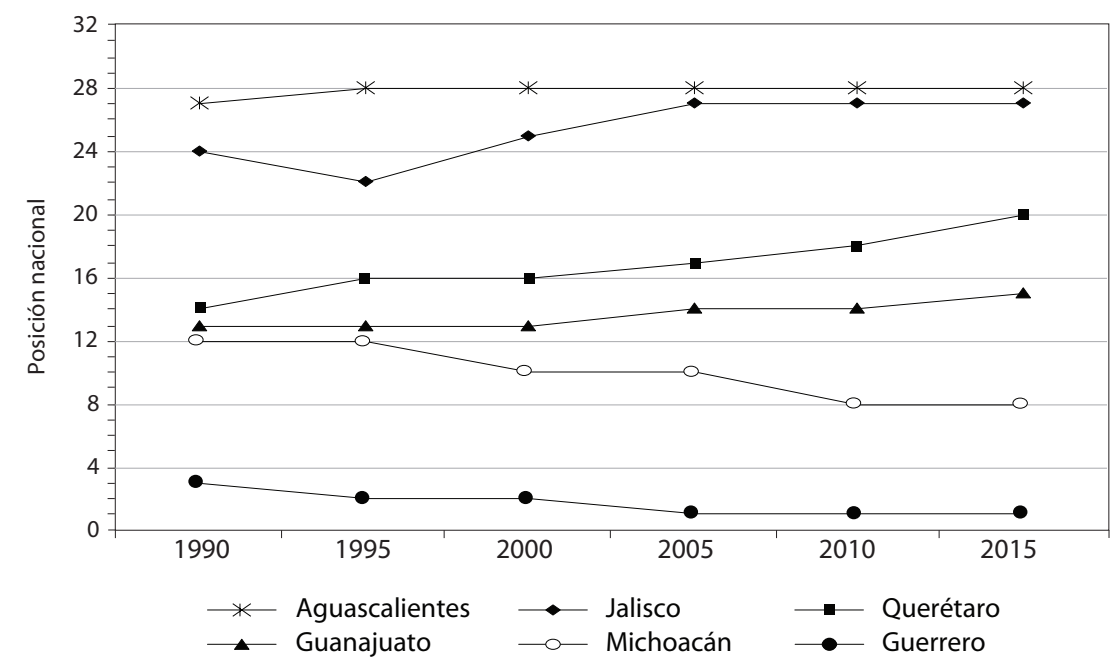

Fuente: Elaboración propia con base en datos de Conapo (2018).

implementó entre 2008 y 2013, y abarcó también parte del gobierno de Fausto Vallejo Figueroa ${ }^{3}$ (2012-2015).

Michoacán es una entidad federativa con marcado rezago socioeconómico e institucional, acentuado en la última década. A diferencia de otros estados del Centro-Occidente de México que han reducido su nivel de marginación, Michoacán muestra un patrón de deterioro social similar al vecino estado de Guerrero (gráfica 1). En 2005, previo a la implementación de la ADLL, Michoacán tenía 37 municipios (32.7\%) con alta y muy alta marginación. Para 2015, Michoacán ocupaba el octavo lugar de mayor marginación del país (Conapo, 2018), con 54 por ciento de población debajo de la línea de pobreza (INEGI, 2016). Los ayuntamientos de la entidad suelen presentar capacidades institucionales bajas, incluso aquellos de marginación media. En particular en los municipios rurales, los funcionarios desarrollan sus funciones con fuerte aislamiento geográfico e institucional; escasos recursos económicos, materiales y humanos; y reducido acceso a la información.

${ }^{3}$ Perteneciente al Partido Revolucionario Institucional (PRI). 


\section{Implementación de la ADLL en Michoacán}

En 2008, el Cedemun convocó a las IEs de la entidad para la implementación del Programa ADll. La Universidad Nacional Autónoma de México (UnAM) Campus Morelia, participó con académicos del Centro de Investigaciones en Geografía Ambiental (CIGA),y del entonces Centro de Investigaciones en Ecosistemas (Cieco). Inicialmente, dos académicos de la Universidad Michoacana de San Nicolás de Hidalgo se integraron al instrumento. Por parte de los gobiernos locales, dos municipios procedieron a la verificación: Morelia (ciudad capital) e Hidalgo (Región Oriente). Con este modesto inicio, el Programa Adll se implementó en Michoacán, para crecer en ańos subsiguientes como un espacio de trabajo en una ICP conformada por el sector gubernamental a nivel estatal (Cedemun-Michoacán), el nivel local (gobiernos municipales) y los expertos universitarios. La interfaz operó durante seis años (2008-2013), siendo una experiencia sin precedentes en la entidad. Durante la implementación del instrumento, la interfaz fue construida y operada por los involucrados sin ningún respaldo conceptual y teórico, y sin ninguna comprensión previa de los factores que operarían en su efectividad.

\section{MÉTODO}

Los estudios sobre la ICP procuran construir una interpretación intersubjetiva y explícita para comprender las características de la interacción entre expertos científicos y decisores, en un contexto determinado. Para ello, se recurre frecuentemente a técnicas de la investigación cualitativa como entrevistas a profundidad, análisis del discurso, grupos focales y observación participante (e.g. White et al., 2008; Nursey-Bray et al., 2014; Sarkki et al., 2014). Esta última ofrece ventajas porque permite recoger datos de las situaciones estando el investigador involucrado en ellas, y siendo el principal instrumento de recopilación de datos (CallejoGallejo, 2002; Kawulich, 2005).

En este estudio, se aplicó el marco de evaluación de la ICP presentado en el diagrama 1. Para cada atributo incluido en dicho marco, se estableció un criterio de evaluación acorde al estudio de caso. La evaluación fue de tipo retrospectivo, y se realizó mediante el análisis interpretativo de la observación participante desde la perspectiva del sector de expertos académicos, guiado por una escala explícita de valoración. El análisis utilizó la evidencia recogida en 49 actividades presenciales de la primera autora entre 2008 y 2013 (cuadro 1), quien fungió como enlace del CIGA-UnAM ante el Cedemun-Michoacán. Otras fuentes de información fueron los trabajos preliminares sobre los resultados e impactos 
CUADRO 1. Tipo y cantidad de actividades de la ADLL en Michoacán entre 2008 y 2013 con participación directa de los autores

\begin{tabular}{lccccccc}
\hline \multicolumn{1}{c}{ Actividad } & 2008 & 2009 & 2010 & 2011 & 2012 & 2013 & Total \\
\hline $\begin{array}{l}\text { Verificaciones a ayuntamientos y elaboración } \\
\text { de recomendaciones }\end{array}$ & 2 & 3 & 6 & 6 & 5 & 5 & 27 \\
$\begin{array}{l}\text { Reuniones de capacitación, planeación y } \\
\text { evaluación entre Cedemun-Michoacán e IES }\end{array}$ & 2 & 2 & 2 & 2 & 2 & 2 & 12 \\
$\begin{array}{l}\text { Capacitaciones ofrecidas a funcionarios } \\
\text { municipales }\end{array}$ & 0 & 0 & 1 & 1 & 3 & 1 & 6 \\
$\begin{array}{l}\text { Actividades en foros nacionales "Agenda } \\
\left.\text { Desde lo Local" } 5^{\circ} \mathrm{y} 7^{\circ}\right)\end{array}$ & 0 & 1 & 0 & 3 & 0 & 0 & 4 \\
\begin{tabular}{l} 
Total \\
\hline
\end{tabular} & 4 & 6 & 9 & 12 & 10 & 8 & 49 \\
\hline
\end{tabular}

Fuente: Elaboración propia.

de la ADLL en la gestión de los municipios michoacanos (Burgos y Paez, 2012), las minutas y recomendaciones emitidas a los ayuntamientos, resultados de encuestas rápidas a funcionarios municipales, y conversaciones entre coautores y colaboradores.

La evaluación se realizó de manera detallada para las dos primeras categorías del marco de evaluación (diagrama 1). Para cada factor y atributos respectivos, se establecieron criterios específicos de evaluación, considerando la naturaleza de la ICP y la mecánica de aplicación del instrumento ADLL. Así, para la categoría de componentes de la interfaz (factores sistémicos) se formularon 15 criterios (cuadro 2), mientras que para la categoría de actitudes de los involucrados (factores actitudinales) se establecieron otros 11 (cuadro 3). Los criterios de evaluación se asociaron a una escala categórica denominada Valor de Consistencia (vc, cuadro 4), la cual refleja, en una escala de 0 a 4 , la frecuencia con la que cada criterio estuvo presente en el conjunto de actividades asistidas por el observador participante (cuadro 1). En la categoría actitudes de los actores, los siete atributos del factor distancia cognitiva-nivel de realidad, y el único atributo del factor autonomía experta (diagrama1), fueron analizados de manera cualitativa a partir de la resignificación de la experiencia del observador participante bajo los criterios de evaluación establecidos (cuadro 3).

El análisis detallado de las dos categorías restantes, i.e. características del conocimiento (factores epistemológicos) y dilemas (factores de conflicto) excede las 
posibilidades de esta publicación. Sin embargo, las características generales observadas en la implementación del instrumento ADLL en Michoacán fueron resumidas en la sección de resultados, enfatizando la importancia de incluir su evaluación sistemática en futuras indagaciones.

CUADRO 2. Criterios de evaluación para los factores y atributos de la categoría de componentes sistémicos de la interfaz

\begin{tabular}{|c|c|c|}
\hline $\begin{array}{l}\text { Factor } \\
\text { sistémico }\end{array}$ & Atributo & Criterio de evaluación \\
\hline \multirow[t]{2}{*}{ Objetivo } & Objeto de frontera (OF) & Claridad en la formulación general del of en el instrumento \\
\hline & Apropiación local del of & Adopción consciente del of por los actores involucrados \\
\hline \multirow[t]{4}{*}{ Estructura } & Mecanismos & Formalidad de los mecanismos de implementación de la interfaz \\
\hline & Participantes & $\begin{array}{l}\text { Cantidad de académicos y ayuntamientos participantes a lo largo } \\
\text { del periodo, en relación con lo requerido }\end{array}$ \\
\hline & Independencia & $\begin{array}{l}\text { Autonomía de las partes expresada en ausencia de intervención, } \\
\text { y no-coerción durante la etapa de verificación }\end{array}$ \\
\hline & Medios & Suficiencia de recursos para cubrir las actividades de la interfaz \\
\hline \multirow[t]{5}{*}{ Procesos } & $\begin{array}{l}\text { Intercambio de } \\
\text { conocimiento }\end{array}$ & Intensidad y frecuencia de flujos bidireccionales de información \\
\hline & $\begin{array}{l}\text { Formación de } \\
\text { capacidades }\end{array}$ & $\begin{array}{l}\text { Realización de actividades de capacitación para académicos y para } \\
\text { funcionarios municipales }\end{array}$ \\
\hline & $\begin{array}{l}\text { Construcción de } \\
\text { confianza }\end{array}$ & $\begin{array}{l}\text { Transparencia en las relaciones entre sectores; trabajo en grupo, } \\
\text { reciprocidad en los compromisos }\end{array}$ \\
\hline & Gestión de conflictos & $\begin{array}{l}\text { Respeto a reglas del programa y a acuerdos preestablecidos entre } \\
\text { las partes }\end{array}$ \\
\hline & Continuidad-iteratividad & Procedimientos para sostener, corregir y adaptar la interfaz \\
\hline \multirow[t]{4}{*}{ Salidas } & Efectividad & Mejoras en la condición inicial del objeto de frontera \\
\hline & Productos & $\begin{array}{l}\text { Cantidad y calidad de recomendaciones para mejorar la gestión } \\
\text { municipal hacia el desarrollo sostenible }\end{array}$ \\
\hline & $\begin{array}{l}\text { Satisfacción de los } \\
\text { participantes }\end{array}$ & Nivel de satisfacción de los involucrados \\
\hline & Cambios actitudinales & $\begin{array}{l}\text { Cambios en las actitudes de expertos y decisores, derivados de su } \\
\text { participación en la interfaz }\end{array}$ \\
\hline
\end{tabular}

Fuente: Elaboración propia. 
CUADRO 3. Criterios de evaluación para los factores y atributos de la categoría actitudes de los involucrados

\begin{tabular}{|c|c|c|}
\hline Factor actitudinal & Atributo & Criterio de evaluación \\
\hline \multirow{7}{*}{$\begin{array}{l}\text { Distancia } \\
\text { cognitiva-niveles } \\
\text { de realidad }\end{array}$} & $\begin{array}{l}\text { Leyes perceptuales de la } \\
\text { realidad }\end{array}$ & Elementos cognitivos para la construcción de la realidad \\
\hline & Pensamiento reflexivo & $\begin{array}{l}\text { Grado de conceptualización y reflexión individual } \\
\text { y colectiva, en torno al objeto de frontera }\end{array}$ \\
\hline & Conectividad informativa & Uso de información externa \\
\hline & Comunicación interna & $\begin{array}{l}\text { Asiduidad del diálogo e interacciones dentro de un mismo } \\
\text { sector }\end{array}$ \\
\hline & $\begin{array}{l}\text { Identificación de } \\
\text { situaciones problemáticas }\end{array}$ & $\begin{array}{l}\text { Amplitud de recursos cognitivos para la identificación } \\
\text { de situaciones problemáticas }\end{array}$ \\
\hline & $\begin{array}{l}\text { Estructuración } \\
\text { de problemas }\end{array}$ & $\begin{array}{l}\text { Nivel de simplificación o complejidad en las relaciones } \\
\text { causales }\end{array}$ \\
\hline & $\begin{array}{l}\text { Construcción de } \\
\text { soluciones }\end{array}$ & $\begin{array}{l}\text { Tipo de soluciones construidas para la atención } \\
\text { de problemas complejos }\end{array}$ \\
\hline Motivación & Apertura-receptividad & Grado de interés de las partes en la interacción \\
\hline Compromiso & $\begin{array}{l}\text { Responsabilidad hacia la } \\
\text { interfaz }\end{array}$ & $\begin{array}{l}\text { Asistencia y cumplimiento de las actividades y tareas } \\
\text { requeridas por la interfaz }\end{array}$ \\
\hline $\begin{array}{l}\text { Percepción } \\
\text { del otro }\end{array}$ & Comprensión de roles & $\begin{array}{l}\text { Aptitudes y habilidades construidas para la construcción } \\
\text { de puentes cognitivos entre sectores }\end{array}$ \\
\hline $\begin{array}{l}\text { Autonomía } \\
\text { experta }\end{array}$ & Control político & $\begin{array}{l}\text { Grado de libertad del sector académico para desplegar } \\
\text { conocimientos y experiencia científica dentro de la interfaz }\end{array}$ \\
\hline
\end{tabular}

Fuente: Elaboración propia.

CUADRO 4. Escala de consistencia para evaluar los atributos de la interfaz ciencia-política, con base en criterios específicos

\begin{tabular}{ccl}
\hline Valor & Consistencia & \multicolumn{1}{c}{ Descripción } \\
\hline 0 & Ausente & Ninguna manifestación del criterio \\
1 & Mala & $\begin{array}{l}\text { Manifestación eventual, rara o errática } \\
\text { Manifestación con cierta recurrencia, sin alcanzar una regularidad } \\
\text { o frecuencia adecuada a las necesidades } \\
\text { Manifestación mayoritaria y dominante del criterio establecido, }\end{array}$ \\
3 & Buenalar & $\begin{array}{l}\text { Con algunas faltantes y altibajos eventuales } \\
\text { Manifestación permanente del criterio establecido }\end{array}$ \\
\hline
\end{tabular}

Fuente: Elaboración propia. 


\section{RESULTADOS}

La aplicación del marco de evaluación (diagrama 1), los criterios específicos (cuadros 2 y 3) y la escala de consistencia (cuadro 4) organizaron la caracterización de la ICP en la implementación de la ADLL 2008-2013 en Michoacán, y dieron rigor a la interpretación del observador participante. En esta sección, los resultados se presentan en tres subsecciones. Las dos primeras presentan resultados detallados de las categorías de componentes sistémicos de la interfaz (factores sistémicos), y actitudes de los involucrados (factores actitudinales). La tercera subsección presenta las principales observaciones sobre el desempeño de la interfaz en las categorías de características del conocimiento (factores epistemológicos), y en los dilemas (factores de conflicto), como aspectos necesarios para una futura indagación.

\section{1) Componentes sistémicos de la interfaz}

Esta categoría, integrada por los factores objetivos, estructura, procesos y salidas de la interfaz, mostró un desempeño entre regular y bueno, con un valor de consistencia

GRÁFICA 2. Valoración de los componentes sistémicos de la interfaz

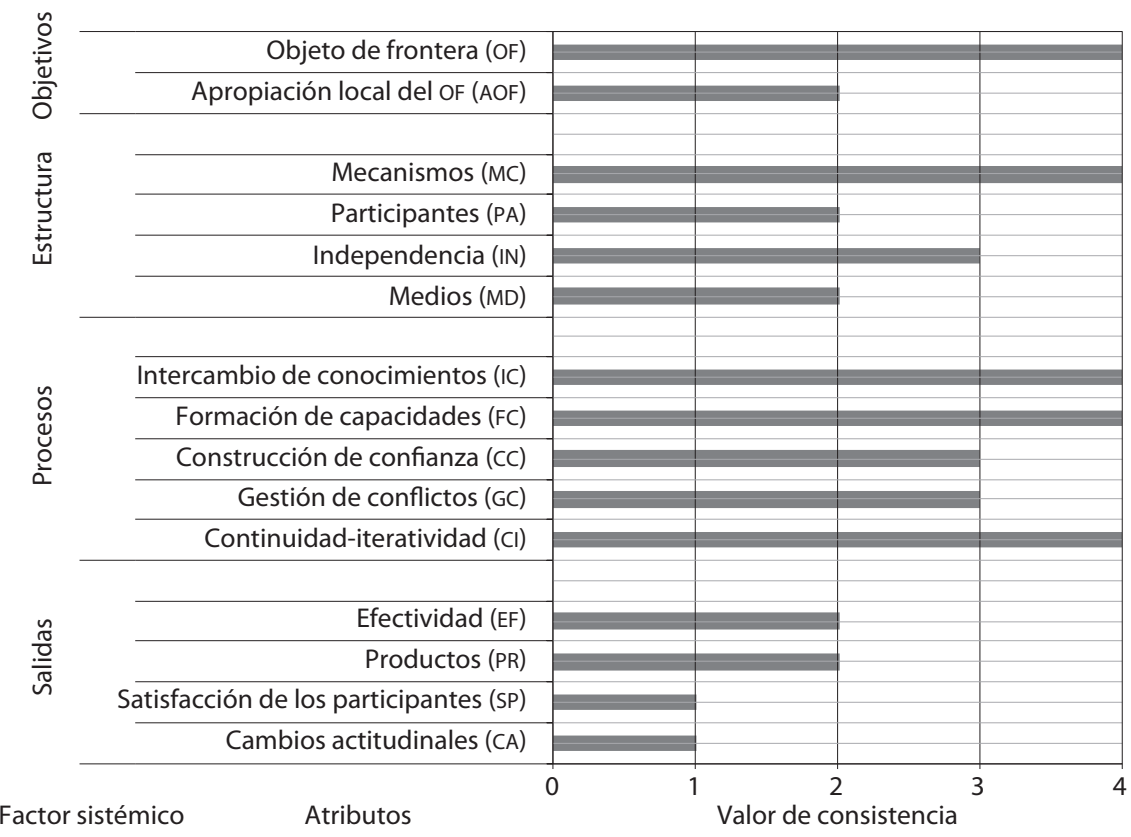

Fuente: Elaboración propia. 
(VC) promedio general $\left(\mathrm{VC}_{\mathrm{CSI}}\right)$ de 2.67 , en la escala de consistencia de 0 a 4 . Según la percepción del observador participante, el factor sistémico mejor posicionado fue el de procesos $\left(\mathrm{VC}_{\mathrm{P}}=3.60\right)$, mientras que el de peor desempeño fue el de salidas $\left(\mathrm{VC}_{S}=1.50\right)$. El desempeño en el factor de objetivos, al igual que en el factor de estructura, fue intermedio, con valores de consistencia de $\mathrm{VC}_{\mathrm{O}}=3.00$ y $\mathrm{VC}_{\mathrm{E}}=2.75$, respectivamente (gráfica 2).

El factor de objetivos de la interfaz mostró un desempeño bueno, pero no excelente $\left(\mathrm{VC}_{\mathrm{O}}=3.00\right)$, debido a la manifestación desigual de sus dos atributos (gráfica 2). Por un lado, el objeto de frontera (of) fue claramente posicionado en el instrumento, siendo la promoción del desarrollo local sostenible el núcleo convocante de la interfaz $\left(\mathrm{VC}_{\mathrm{of}}=4\right.$; gráfica 2$)$. Sin embargo, durante la implementación, la apropiación local del of mostró debilidades $\left(\mathrm{VC}_{\mathrm{aof}}=2\right)$. Si bien la relación entre la Agenda 21 global y la ADLL en México era señalada en las actividades de inducción al programa por los funcionarios estatales ( $\mathrm{FE}$ ), dicha relación quedaba diluida durante las actividades de verificación y de capacitación. Así, tanto expertos (Ex) como funcionarios municipales (FM) frecuentemente perdían la visión estratégica detrás de la construcción de la interfaz. La inconsistencia entre la fortaleza institucional del of a nivel nacional y la debilidad de apropiación a nivel local restó impacto al instrumento.

El factor sistémico de estructura mostró un VC promedio entre regular y bueno $\left(\mathrm{VC}_{\mathrm{E}}=2.75\right.$; gráfica 2$)$. El atributo de mecanismos mostró gran fortaleza $\left(\mathrm{VC}_{\mathrm{mc}}=4\right)$, dado que los procedimientos para construir la interfaz estaban claramente especificados en los lineamientos del instrumento. Además, estos fueron celosamente resguardados por los FE del Cedemun. El desempeńo excelente de los mecanismos se reflejó en el buen desempeño del atributo de independencia $\left(\mathrm{VC}_{\mathrm{in}}=3\right)$. En general, la relación entre FM y EX sostuvo una alta autonomía de las partes, pero hubo episodios aislados de coerción por parte de los ayuntamientos para que las IEs volcaran sus dictámenes a su favor cuando el municipio se encontraba cerca de cumplimentar los requisitos para ser premiado como Buen Gobierno Municipal. Un desempeño menos consistente se observó en los atributos de participantes $\left(\mathrm{VC}_{\mathrm{pa}}=2\right)$ y medios $\left(\mathrm{VC}_{\mathrm{md}}=2\right)$. De acuerdo con datos del Cedemun (2013), la participación de los ayuntamientos incrementó a partir de 2010, y alcanzó en 2013 un máximo de 55 ayuntamientos verificados (gráfica 3 ), equivalente a 46.9 por ciento de los 113 municipios michoacanos. La incorporación de IES y verificadores se incrementó gradualmente, y alcanzó en ese mismo año 11 instituciones académicas y 134 verificadores activos. Sin embargo, la cantidad de Ex involucrados no estuvo a la altura de las necesidades. Similar- 
GRÁFICA 3. Participación de ayuntamientos e Instituciones de Educación Superior (IES) en el Programa ADLL 2008-2013 en Michoacán

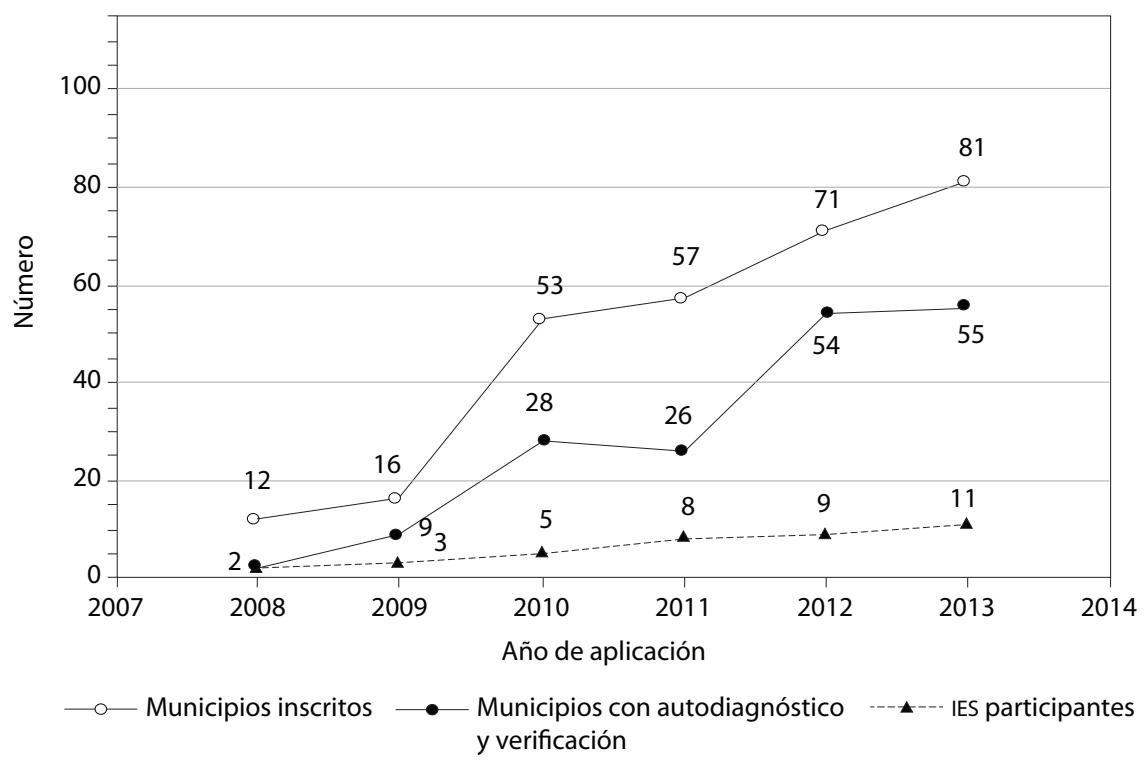

Fuente: Elaboración propia con base en datos del Cedemun (2013).

mente, las limitaciones en los medios disponibles reflejaron el deterioro financiero del estado de Michoacán. El personal del Cedemun asignado al programa fue escaso durante todo el periodo 2008-2013. El traslado a cada punto de la geografía michoacana se realizaba en vehículos del Cedemun, aunque fueron los propios ayuntamientos e IES quienes solventaron gastos de alimentos, hospedaje y papelería. A medida que se incrementó el número de ayuntamientos e IES participantes, se redujeron los fondos para su implementación. Esta restricción económica operó como una traba para la incorporación al programa de aquellos ayuntamientos rurales más distantes de la ciudad capital, y con restricciones presupuestarias para subsanar incluso gastos menores de su funcionamiento. Como resultado de las limitaciones en participantes y medios, la cobertura de municipios del programa ADLL en Michoacán quedó lejos de aquella alcanzada en otros estados del Centro-Occidente de México, como Jalisco, Querétaro, Guanajuato y Aguascalientes, donde se cubrió 100 por ciento de municipios. En esos casos, algunos gobiernos estatales como Jalisco establecieron estrategias particulares y 
fondos suficientes para lograr dicha cobertura. En otros, como Aguascalientes, la reducida extensión del territorio estatal y del número de municipios facilitó la implementación.

El factor sistémico de procesos mostró el mejor desempeño $\left(\mathrm{VC}_{\mathrm{p}}=3.60\right)$, debido a la consistencia buena y excelente de sus cinco atributos (gráfica 2). La experiencia inicial en 2008, si bien pequeña en número de participantes (gráfica 3), fue estimulante para todos los involucrados. El Cemedun y las IEs imprimieron a la ADLL en Michoacán un sello que fue distintivo en el país. Aun sin un adecuado respaldo conceptual y teórico de las características de las ICPs, el Programa ADLL fue concebido empíricamente como una interfaz, en tanto oportunidad para acercar el conocimiento y experiencia científico-académica a los funcionarios de los ayuntamientos michoacanos, brindar asesoría y escuchar las problemáticas locales de la voz directa de los protagonistas. Las IEs acordaron la realización de las verificaciones in situ (en cada cabecera municipal), para tener contacto cara a cara con cada FM y su entorno. Cada verificación demandó el traslado de los EX hasta cada cabecera y largas jornadas de trabajo personalizado, un esquema sostenido durante seis años. Este procedimiento promovió un interesante y productivo intercambio de conocimientos $\left(\mathrm{VC}_{\mathrm{ic}}=4\right.$; gráfica 2$)$. Las IES acordaron maximizar esfuerzos en la emisión escrita de recomendaciones para alimentar los planes de mejora municipales; ello requería trabajo posterior para integrar información, justificar y emitir sugerencias. El atributo de formación de capacidades mostró también un excelente desempeño $\left(\mathrm{VC}_{\mathrm{fc}}=4\right)$. El Cedemun realizó anualmente reuniones y talleres de capacitación para EX y FM, y también articuló actividades de capacitación entre estos (cuadro 1). Al menos dos veces al ańo se sostuvieron reuniones entre Cedemun e IES para la planificación y evaluación del ejercicio. Las IEs fueron invitadas a participar en los Foros Nacionales de la ADLL y a escuchar presentaciones de presidentes municipales de todo el país. Las relaciones sostenidas entre los EX y FE construyeron vínculos de confianza entre las partes, si bien esta no alcanzó su valor máximo de consistencia $\left(\mathrm{VC}_{\mathrm{cc}}=3\right)$, debido a episodios eventuales de dudas entre sectores no resueltas adecuadamente. En correlación, el atributo de gestión de conflictos mostró un desempeño consistente, pero con altibajos esporádicos $\left(\mathrm{VC}_{\mathrm{gc}}=3\right)$. La forma de trabajo en Michoacán promovió la continuidad-iteratividad de la interfaz durante los seis años $\left(\mathrm{VC}_{\mathrm{ci}}=4\right)$. Cada año las IEs y el Cedemun discutieron largamente los criterios técnicos y éticos de las verificaciones, y realizaron ajustes para adaptar la implementación a las circunstancias presentes en la entidad, al menos en aquellos aspectos del instrumento que podían ser mejorados a nivel estatal. 
Finalmente, el factor de salidas fue el más débil de los componentes sistémicos, porque el instrumento no contempló en su diseño mecanismos adecuados para dar seguimiento a la efectividad, productos, efectos e impactos de la interfaz. La ADLL medía su éxito por el número de municipios e indicadores con certificados verdes, lo cual señalaba de manera indirecta y difusa los cambios logrados en la gestión municipal para avanzar hacia el desarrollo sostenible, es decir, la efectividad de la interfaz sobre el objeto de frontera $\left(V_{\mathrm{ef}}=2\right.$; gráfica 2$)$. Del total de ayuntamientos participantes, solo una pequeña proporción mejoró sus procesos de gestión para el desarrollo sostenible durante cortos periodos. La interfaz fue más efectiva en los municipios urbanos, donde las mejoras se sostuvieron solamente dentro de un mismo mandato de gobierno. La mayor parte de municipios participantes fueron rurales, y en ellos la ADLL mostró mejoras pequeñas o nulas. A modo de ejemplo, Burgos y Paez (2012) documentaron el magro impacto de la ADLL en la gestión local en materia ambiental. El contacto puntual una vez al año entre sectores - si bien activó los flujos de conocimiento-, no fue suficiente para enriquecer la toma de decisiones con conocimiento científico durante todo el ańo y asegurar cambios permanentes en la calidad de la gestión. Si bien se generaron productos concretos con las recomendaciones de los EX a los ayuntamientos luego de cada verificación anual, estos productos fueron puntuales y acotados; $\mathrm{y}$ no fueron complementados con información pertinente y útil durante el resto del año $\left(\mathrm{VC}_{\mathrm{pr}}=2\right)$. Por último, los atributos de satisfacción de los participantes y de cambios actitudinales fueron, en opinión del observador participante, poco consistentes a lo largo del periodo evaluado, con valores de $\mathrm{VC}_{\mathrm{sp}}=1$ y $\mathrm{V}_{\mathrm{ca}}=1$, respectivamente. Esto se reflejó, por ejemplo, en cambios menores en la motivación e involucramiento de los ayuntamientos para la adopción del instrumento y en el incremento modesto de expertos verdaderamente comprometidos con la interfaz. La evaluación de estos atributos se complementa en la siguiente categoría.

\section{2) Actitudes de los involucrados}

Los cinco factores y once atributos de esta categoría (diagrama 1) reflejaron los aspectos actitudinales que dan vida o asfixian la construcción de una ICP. En esta evaluación, los criterios específicos del cuadro 4 fueron esenciales para orientar al observador participante en el análisis cualitativo y en la resignificación de su experiencia.

La exploración del factor distancia cognitiva-niveles de realidad, con sus siete atributos (diagrama 1), reveló a detalle las dificultades cognitivas implicadas en la construcción de la interfaz. El cuadro 5 muestra los atributos que explican la 


\section{CUADRO 5. Valoración cualitativa de los siete atributos del factor distancia cognitiva-niveles de realidad}

\begin{tabular}{|c|c|c|}
\hline Atributo & Funcionarios municipales & $\begin{array}{c}\text { Expertos } \\
\text { (académicos y estudiantes de posgrado) }\end{array}$ \\
\hline $\begin{array}{l}\text { Leyes } \\
\text { perceptuales } \\
\text { de la realidad }\end{array}$ & $\begin{array}{l}\text { Percibida de manera individual a partir de } \\
\text { vivencias personales y relaciones cotidianas, } \\
\text { con escaso grado de abstracción. Escasa } \\
\text { articulación con conceptualizaciones } \\
\text { provenientes de fuentes externas. Poco } \\
\text { contacto con las visiones de otros } \\
\text { observadores, aun con otros pares de la } \\
\text { función municipal. }\end{array}$ & $\begin{array}{l}\text { Idealizada, abstracta, vista a través de } \\
\text { modelos y conceptos construidos por la } \\
\text { comunidad científica principalmente } \\
\text { internacional, con escaso contacto directo } \\
\text { con las situaciones problemáticas } \\
\text { cotidianas y las visiones de otros } \\
\text { observadores más allá de la academia. }\end{array}$ \\
\hline $\begin{array}{l}\text { Pensamiento } \\
\text { reflexivo }\end{array}$ & $\begin{array}{l}\text { Poco desarrollado y poco estimulado por las } \\
\text { estructuras de gobierno, en atención } \\
\text { a la urgencia, el tiempo escaso y la } \\
\text { necesidad de acciones concretas e } \\
\text { inmediatas. }\end{array}$ & $\begin{array}{l}\text { Desarrollado, pero encapsulado en el seno } \\
\text { del sector académico, con pares } \\
\text { principalmente de la misma especialidad. }\end{array}$ \\
\hline $\begin{array}{l}\text { Conectividad } \\
\text { informativa }\end{array}$ & $\begin{array}{l}\text { Escaso acceso y procesamiento de } \\
\text { información externa. La información } \\
\text { proviene fundamentalmente de fuentes } \\
\text { gubernamentales (programas, } \\
\text { instituciones). }\end{array}$ & $\begin{array}{l}\text { Amplio acceso a información externa } \\
\text { proveniente de múltiples fuentes. Se } \\
\text { preconcibe la confiabilidad de las fuentes } \\
\text { de acuerdo con criterios científicos. }\end{array}$ \\
\hline $\begin{array}{l}\text { Comunicación } \\
\text { interna }\end{array}$ & $\begin{array}{l}\text { Fuertes dificultades para el diálogo entre } \\
\text { áreas del ayuntamiento. Escasa visualización } \\
\text { de su importancia, sin iniciativas para } \\
\text { promoverla. Cultura burocrática. }\end{array}$ & $\begin{array}{l}\text { Visualizada como fundamental hacia las } \\
\text { funciones de gobierno. Muy practicada } \\
\text { entre pares académicos. }\end{array}$ \\
\hline $\begin{array}{l}\text { Identificación de } \\
\text { situaciones } \\
\text { problemáticas }\end{array}$ & $\begin{array}{l}\text { Lectura directa y desestructurada de una } \\
\text { realidad construida por la cotidianidad del } \\
\text { sujeto-observador. }\end{array}$ & $\begin{array}{l}\text { Lectura indirecta, fuertemente } \\
\text { estructurada y posiblemente rígida, bajo } \\
\text { conceptos, datos e interpretaciones } \\
\text { basadas en modelos mentales } \\
\text { científicamente concebidos. }\end{array}$ \\
\hline $\begin{array}{l}\text { Estructuración } \\
\text { de problemas }\end{array}$ & $\begin{array}{l}\text { Fuertemente basada en la experiencia } \\
\text { cotidiana, con pocos conceptos e } \\
\text { información disponible; apremiada por la } \\
\text { necesidad y urgencia de resolverlos. }\end{array}$ & $\begin{array}{l}\text { Basada exclusivamente en la visión del } \\
\text { sector científico; excesiva abstracción; } \\
\text { condicionada por prejuicios disciplinarios. }\end{array}$ \\
\hline $\begin{array}{l}\text { Construcción } \\
\text { de soluciones }\end{array}$ & $\begin{array}{l}\text { Simplificantes, incompletas; diseñadas para } \\
\text { su ejecución en tiempos extremadamente } \\
\text { rápidos, a bajo costo. Enmascaran la } \\
\text { complejidad del problema inicial. }\end{array}$ & $\begin{array}{l}\text { Dominada por una lógica que no } \\
\text { incorpora las restricciones existentes. Sin } \\
\text { apremio ni urgencia por diseñar } \\
\text { soluciones a corto plazo. Poco aplicables, } \\
\text { costosas, requieren mucho tiempo y } \\
\text { personal para su implementación. }\end{array}$ \\
\hline
\end{tabular}

Fuente: Elaboración propia. 
gran distancia cognitiva y el contraste de niveles de realidad entre los FM y EX. Desde la perspectiva del observador participante, se detectó que las leyes perceptuales de la realidad, es decir, la forma en que la realidad es percibida y construida por cada sector, están ubicadas en extremos de un gradiente entre conocimiento empírico y conocimiento abstracto. Por ejemplo, el atributo de pensamiento reflexivo en torno al objeto de frontera - i.e. la gestión para el desarrollo sostenible- reflejó dos mundos muy diferenciados (cuadro 5). En el caso de los FM, las urgencias de las decisiones cotidianas, pero también la falta de visión, marcos conceptuales y heurísticos, parecen acotar las oportunidades para el pensamiento reflexivo sobre la gestión. Además, los FM de los municipios michoacanos mostraron escasa conectividad informativa, debido al poco acceso o consulta de información externa. Ello se conjunta con una insuficiente comunicación interna entre áreas del mismo ayuntamiento. Los flujos de información limitados contraen las posibilidades de reflexión colectiva sobre la gestión. En los Ex, los mismos atributos de pensamiento reflexivo, conectividad informativa y comunicación interna están ampliamente desarrollados. Sin embargo, estos asumen, en general, que dichos atributos deberían estar bien desarrollados también entre los FM sin reconocer las restricciones para desplegarlos en los contextos locales.

Por otra parte, los tres atributos relacionados con la identificación, estructuración de problemas y construcción de soluciones como componentes del nivel de realidad mostraron interesantes contrastes (cuadro 5). Mientras los FM identifican, estructuran y construyen potenciales soluciones con base en su experiencia individual y cotidiana, con poco contacto con otras experiencias, incluidas las de sus pares, los ex problematizan y crean soluciones más allá de su experiencia individual y cotidiana, con soporte en experiencias abstractas y lejanas. Esto fomenta brechas en la comprensión del otro, que conducen a la descalificación mutua. En general, el factor de distancia cognitiva-niveles de realidad en sus siete atributos muestra mayores contrastes entre FM sin formación profesional - una situación común en la mayor parte de los municipios michoacanos- y aquellos EX menos involucrados en la interfaz — una situación frecuente en el sector académico.

La exploración de los factores de motivación, compromiso y percepción del otro - y sus atributos de apertura-receptividad, responsabilidad y comprensión de roles, respectivamente- detectó la actitud general de cada uno de los tres sectores (FE, FM y EX) ante la interfaz construida en la $\mathrm{ADLL}_{2008-2013}$. Considerando el VC promedio por sector de los tres atributos (gráfica 4), el mejor desempeño fue mostrado por los FE del Cedemun $\left(\mathrm{VC}_{\mathrm{FE}}=3.67\right)$. En contraste, los FM mostraron 
GRÁFICA 4. Valor de consistencia (vc) en los atributos actitudinales de los actores involucrados en la interfaz

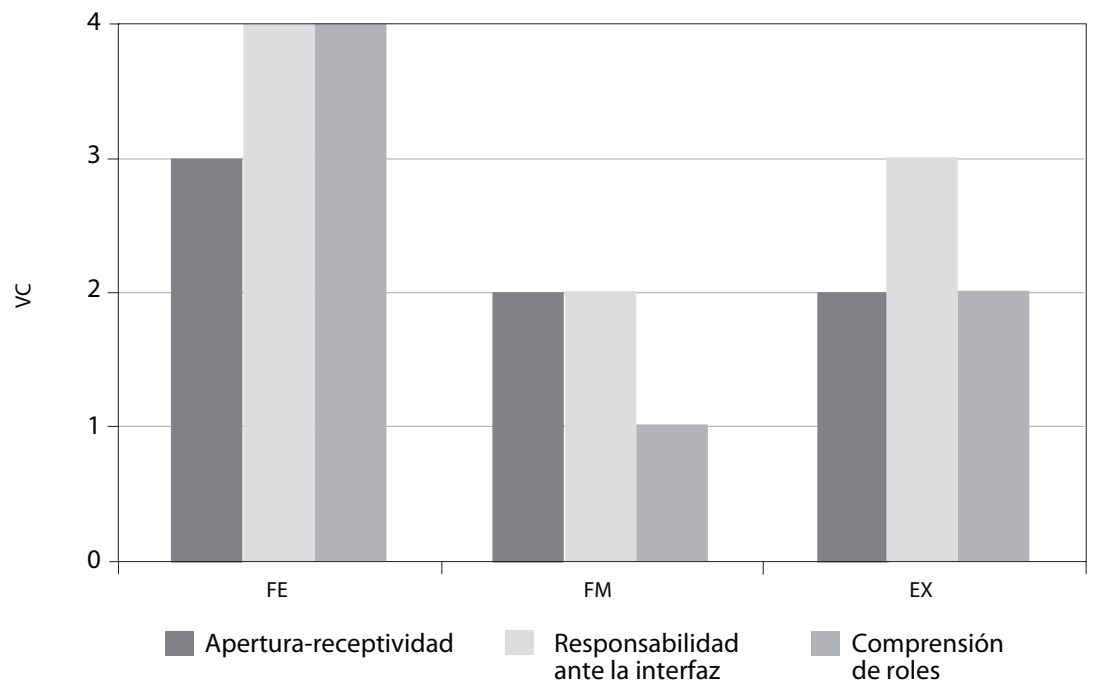

Fuente: Elaboración propia. FE: funcionarios estatales del Cedemun-Michoacán; FM: funcionarios municipales; EX: expertos académicos.

actitudes poco consistentes $\left(\mathrm{VC}_{\mathrm{FM}}=1.67\right)$ entre municipios y a lo largo del periodo evaluado, mientras que los Ex reflejaron actitudes con una consistencia moderada $\left(\mathrm{VC}_{\mathrm{E}}=2.33\right)$.

El factor actitudinal de motivación y su atributo de apertura-receptividad (ar), tuvo en los FE el mejor exponente, porque estos mantuvieron un papel dinámico y abierto en la articulación de la interfaz hacia todos los involucrados, aunque con eventos ocasionales de mayor rigidez $\left(\mathrm{VC}_{\mathrm{FE}-\mathrm{ar}}=3\right.$; gráfica 4$)$. En cambio en los $\mathrm{FM}$, la apertura-receptividad fue muy variable entre ayuntamientos $\left(\mathrm{VC}_{\mathrm{FM}-\mathrm{ar}}=2\right)$. En algunos, la interacción con los Ex era esperada con interés y entusiasmo, mientras que en otros resultaba un momento ajeno y forzado. Similar respuesta mostró la apertura-receptividad de los $\mathrm{EX}\left(\mathrm{VC}_{\mathrm{EX}-\mathrm{ar}}=2\right)$, porque solo un grupo reducido de académicos mostró alta constancia e interés a lo largo de todo el periodo, y la mayoría fue participante eventual con escasa apertura-receptividad y baja permanencia.

El factor de compromiso y su atributo de responsabilidad (r) ante la interfaz mostró un desempeńo destacado en los FE. El impulso del instrumento por los 
FE del Cedemun fue muy constante, aun con el cambio de gobierno y partido político entre los periodos 2008-2012 y 2012-2013 $\left(\mathrm{VC}_{\mathrm{FE}-\mathrm{r}}=4\right.$; gráfica 4). Pero los FM mostraron una consistencia muy irregular y contrastante entre municipios inscritos en el programa $\left(\mathrm{VC}_{\mathrm{FM}-\mathrm{r}}=2\right)$. Varios ayuntamientos se involucraron en los procesos de verificación con absoluta responsabilidad, lo cual se manifestó en el cuidado y dedicación prestados a la preparación de documentos y espacios físicos e incluso en la creación de momentos de convivencia entre participantes. Pero otros ayuntamientos inscritos mostraron mínima responsabilidad, con displicencia en la preparación de documentos y escasa atención a las necesidades de la interacción. Finalmente, entre los ex el atributo de responsabilidad tuvo una consistencia buena $\left(\mathrm{VC}_{\mathrm{EX}-\mathrm{r}}=3\right)$, dado que las IEs involucradas encontraron mecanismos para responder ante los compromisos adquiridos, sea incorporando estudiantes de posgrado o redoblando esfuerzos individuales.

En cuanto al factor percepción del otro, y el atributo comprensión de roles (cr), los FE mostraron un desempeño excelente ( $\mathrm{VC}_{\mathrm{FE}-\mathrm{cr}}$; gráfica 4). El Cedemun-Michoacán contó en ambos periodos con funcionarios de alto perfil técnico en temas de municipalismo, con interés hacia el conocimiento técnico y preparación en el ámbito académico. Este perfil propició que los FE se movieran con gran comprensión de roles hacia ambos lados de la interfaz. En contraste, los FM mostraron muy baja consistencia en dichas competencias $\left(\mathrm{VC}_{\mathrm{FM}-\mathrm{cr}}=1\right)$. En la mayor parte de ayuntamientos, particularmente en aquellos rurales, los FM no contaban con experiencia previa de contacto con Ex, y la comprensión del rol de la actividad académica fue limitada. Por último, entre los Ex la comprensión de los roles de los FE y FM fue variable a lo largo de la implementación del instrumento $\left(\mathrm{VC}_{\mathrm{EX}-\mathrm{cr}}=2\right)$. $\mathrm{Al}$ inicio, los Ex no contaban con la sensibilidad para comprender los procesos de gestión; sin embargo, el Cedemun y las propias IEs fomentaron actividades de capacitación en temas de municipalismo. Por ello, una parte de los Ex mejoraron su comprensión de los roles y de las competencias que atañen a los FM.

El último factor actitudinal es la autonomía experta, cuyo único atributo considerado es el control político de la interfaz (cuadro 1). El sector académico de Michoacán sostuvo interacciones frecuentes con el nivel de gobierno estatal y municipal para la implementación del instrumento durante el periodo 2008-2013, pero también se vinculó con el nivel federal a través de un representante académico del estado en el Consejo Nacional desde lo Local para el diseño del instrumento. La autonomía experta, entonces, puede valorarse respecto a los tres niveles gubernamentales. Durante la implementación de la ADLL, los mecanismos y reglas de trabajo estaban prefijados, de modo que, a priori, la autonomía experta era 
baja. Sin embargo, el sector académico en Michoacán tuvo la libertad de agregar criterios y matices en la implementación en el estado, bajo un control político moderado y razonable por parte del nivel estatal. Esta autonomía moderada en la implementación dio riqueza y adaptabilidad al instrumento para atender las condiciones particulares de los municipios michoacanos. En cuanto a los ajustes al diseño del instrumento, la representación académica ante el órgano federal del Consejo Nacional Desde lo Local constituyó una vía abierta e independiente con alta autonomía experta para sugerir e introducir mejoras en sucesivas versiones durante el periodo evaluado. El patrón de moderada autonomía experta en la implementación y de alta autonomía en el diseño de los instrumentos de política pública favorece la calidad técnica de los instrumentos.

\section{3) Características del conocimiento y dilemas}

El desarrollo a profundidad de la categoría de características del conocimiento, con sus tres factores epistemológicos y sus ocho atributos; así como la categoría de dilemas, con sus cuatro factores y cinco atributos (diagrama 1), excede con creces las posibilidades de este texto. Sin embargo, la importancia de estas categorías demanda la presentación de algunos comentarios generales que complementen esta evaluación y orienten una futura indagación.

En relación con las características del conocimiento implicado en la ICP y los factores de relevancia, confiabilidady legitimidad, cabe señalar que desde el inicio del instrumento ADLL en Michoacán, los EX asumieron la importancia de proveer información sólida y confiable a los FM. Para ello, la distribución de municipios por verificar respondió a las regiones geográficas donde cada IEs tenía mayores investigaciones y conocimiento local. De igual modo, los temas para el desarrollo sostenible del instrumento fueron distribuidos según las capacidades disciplinarias de cada experto. Sin embargo, en ningún momento se establecieron mecanismos internos de revisión por pares o de autoevaluación cruzada para supervisar los atributos de rigor, comunicación de la incertidumbre y diversidad de perspectivas en el conocimiento comunicado. Tampoco se crearon instancias de debate académico para dirimir aspectos conceptuales y teóricos sobre los temas críticos del instrumento, y sobre sus particularidades en los contextos locales. Otra debilidad muy preocupante fue la ausencia de mecanismos de levantamiento y registro de las opiniones de los FM sobre la calidad y utilidad del conocimiento comunicado por los Ex.

En cuanto a los dilemas y sus factores de conflicto, la implementación de la ADLL en Michoacán se enfrentó a situaciones recurrentemente presentes en 
la construcción de ICPs (diagrama 1), pero sin mecanismos para su reducción. El dilema de agenda individual vs agenda común fue afectado por la cultura académica vigente en México, dominada por el bajo interés personal de los investigadores hacia actividades fuera del ámbito académico. El instrumento no consideró en su diseño un mecanismo de reconocimiento a la labor de las IES y sus académicos, lo que operó en contra de un mayor involucramiento. Los dilemas de simplicidad vs complejidad, y rapidez vs profundidad del conocimiento comunicado no fueron suficientemente visualizados como problemas, por lo que no fueron atendidos de manera colectiva por los ex. Estos dilemas están íntimamente relacionados con temas de rigor científico y de actualización del conocimiento comunicado, y requieren estrategias interdisciplinarias con un buen trabajo en equipo, asi como síntesis de datos, información y conocimientos.

Finalmente, el dilema del conocimiento por oferta vs por demanda no se expresó de manera evidente en el caso de la ADLL, dado que la interfaz se creó para la fase de implementación de un instrumento de política pública ya establecido. Los EX se abocaron a proveer conocimiento sobre los temas de gestión local hacia la sostenibilidad contenidos en el instrumento, i.e. conocimiento por demanda. Las urgencias por cumplir con la implementación del instrumento redujeron las oportunidades para que los Ex pudieran ofertar otros conocimientos científicos pertinentes a los ayuntamientos, derivados de investigaciones originales en cada región de Michoacán. Un mejor balance del flujo de conocimientos por oferta y por demanda pudo haber expandido los alcances de la interfaz.

\section{DISCUSIÓN Y CONCLUSIONES}

Este estudio empírico de tipo retrospectivo es una contribución al diseño de instrumentos políticos para propiciar el encuentro entre la ciencia y la política, a nivel de los gobiernos locales. Hasta el momento, este es un campo de estudio poco desarrollado el cual, sin embargo, es altamente necesario para mejorar los procesos de descentralización que ubican al municipio como un actor central del desarrollo local. En América Latina, estos procesos han mostrado rezagos, están inacabados y presentan importantes deficiencias (Rosales, 2012). De igual modo en México, los gobiernos locales, particularmente en municipios rurales, no logran construir capacidades institucionales para enfrentar los problemas de alta complejidad en sus territorios (Cabrero-Mendoza, 2004; Sosa-López, 2012), pero tampoco cuentan con asistencia externa para subsanar dichas deficiencias. Frente a estas condiciones, la creación de interfaces efectivas entre expertos académicos y funcionarios municipales es una necesidad urgente, que se agudiza ante las metas de los 
Objetivos del Desarrollo Sostenible planteados para 2030 (Slack, 2015; Fenton y Gustafsson, 2017; Ojeda-Medina, 2020).

La versión mexicana de Agenda Local 21 implementada en Michoacán entre 2008 y 2013 propició la creación de la ICP local entre funcionarios y expertos académicos, y constituyó una experiencia única de alto valor político, científico y humano para quienes se involucraron de manera activa. Lamentablemente, la experiencia no contó en su momento con un respaldo conceptual y teórico sobre la construcción y efectividad de las ICPs como el presentado en este trabajo. Por ello, el marco de evaluación aquí propuesto fue valioso y necesario para dar orden y rigor al análisis semicuantitativo y cualitativo de tipo retrospectivo, y rescatar lecciones aprendidas para el diseño de futuros instrumentos de política pública tendientes a la creación de estas interfaces. Las cuatro categorías analíticas, sus factores y atributos, así como la escala de consistencia fueron elementos esenciales para reducir y hacer explícitos los sesgos subjetivos inherentes a estudios altamente cualitativos. El uso de este marco en su modo ex ante permitirá a académicos y funcionarios tanto mejorar el diseño de políticas públicas e instrumentos, como elevar la efectividad de las interfaces al momento de su implementación.

Entre los componentes sistémicos, la evaluación de la ADLL en Michoacán demostró la relevancia de establecer con claridad el objetivo de la interfaz y su objeto de frontera, así como asegurar la fuerte apropiación por las partes involucradas. Las investigaciones especializadas insisten en la relevancia del objeto de frontera en la construcción de ICPs efectivas, debido a su capacidad para articular sistemas de conocimiento en comunidades diferenciadas (Star, 2010; Steger et al., 2018). De igual modo, los factores estructurales para crear ICPs deben ser considerados adecuadamente en el diseño de instrumentos y en su correcta implementación (Sarkki et al., 2015). En la ADLL en Michoacán, los factores estructurales mostraron fortalezas debido a las capacidades técnicas disponibles, motivación, compromiso y comprensión de roles, por parte de los funcionarios estatales encargados de la implementación. Sin embargo, el instrumento no contempló estímulos o incentivos para motivar el involucramiento y el compromiso de los protagonistas de la interfaz, i.e. funcionarios municipales y expertos académicos. Aún prevalece una escasa cultura de comunicación y colaboración entre ciencia y política en México, así como en la mayor parte de países del mundo (Swilling, 2014; More, 2019). Por ello, a futuro es esencial incluir mecanismos para la promoción y adopción de la ICP por las partes. Por último, las salidas de la interfaz fueron el componente sistémico más débil de la ADLL en Michoacán. Las salidas reflejan los impactos de la interacción, pero son difícilmente visibles 
o evaluables en el corto plazo. Por ello, los instrumentos políticos que promueven ICP deberían incluir mecanismos de seguimiento muy concretos, que permitan identificar los productos y efectos inmediatos de la interacción, mediante indicadores, medidas de satisfacción, espacios de reflexión y evaluaciones periódicas intersectoriales, entre otros recursos. Cabe recordar que las salidas operan como bucles de retroalimentación e impactan sobre la propia interfaz, incidiendo también en las actitudes de los involucrados (Cash et al., 2002).

El papel de las actitudes de los involucrados aparece recurrentemente como un tema subyacente a la efectividad de la ICP (Engels, 2005; Augsburg, 2014). El estudio de caso detectó una gran distancia cognitiva y niveles de realidad muy diferenciados entre expertos académicos y funcionarios municipales. Howart y Painter (2016) señalaron esta misma condición de alta distancia cognitiva entre la visión de los reportes del IPCC y aquella de los gobiernos locales, lo que ha creado barreras para diseminar la problemática del cambio climático entre los funcionarios municipales. En aras de lograr mayor efectividad de las ICP es imperioso definir medios y mecanismos creativos para fomentar una cultura institucional basada en la tolerancia, apertura y respeto mutuo entre expertos científicos y hacedores e implementadores de políticas en los niveles locales de gobierno.

Este estudio empírico indagó de manera limitada en las categorías de características del conocimiento (factores epistemológicos) y dilemas (factores de conflicto) en la implementación de la ADLL en Michoacán. Sin embargo, se detectaron algunas deficiencias del instrumento para asegurar la confiabilidad del conocimiento comunicado, y para reducir los dilemas detrás de la producción y comunicación del conocimiento experto a funcionarios. El caso ilustró los desafíos y cambios requeridos en la práctica científica para que el conocimiento científico producido sea útil, oportuno y adecuado para el diseńo, la implementación y la evaluación de políticas públicas y sus instrumentos. La práctica académica requerirá nuevos estándares y criterios para implementar verdaderos proyectos de investigación transdisciplinaria con rigor científico. Este es actualmente la mejor vía para conjuntar la ciencia, la política y la sociedad (Klein et al., 2001), y en esta tarea las agencias que regulan la producción científica del país tienen un papel central.

Además del estudio empírico, este trabajo propuso un marco de evaluación de factores determinantes en la ICP, que responde al estado del arte en la materia. Ciertamente, la problemática ambiental y los retos de la sostenibilidad ocupan un lugar central en el interés y estudio de estas interfaces. Sin embargo, este marco de evaluación puede dar soporte metodológico para el diseńo de futuros 
instrumentos de política pública orientados a crear interfaces entre expertos y decisores en cualquier nivel de organización y en cualquier ámbito de la política pública. Un campo de aplicación es el diseño de políticas en innovación, cuyo punto central es la creación de redes y sistemas interactorales capaces de intercambiar conocimientos para agregar valor económico y social en territorios y regiones específicas.

Finalmente, la activación de la interfaz ciencia-política es un asunto que requiere en México una pronta atención. Para que ello ocurra, es fundamental comprender los factores institucionales, epistemológicos, actitudinales y operativos que usualmente operan como barreras para disociar las formas de pensamiento propias de cada sector. Queda en manos de las instituciones académicas y científicas, por un lado, y de los hacedores de políticas públicas, por otro, hacer apuestas mayores para superarlas. GGEิ

\section{REFERENCIAS}

Augsburg, T. (2014), "Becoming Transdisciplinary: The Emergence of the Transdisciplinary Individual”, World Futures, 70(3-4), pp. 233-247.

Broström, A. y M. McKelvey (2018), "Engaging Experts: Science-policy Interactions and the Introduction of Congestion Charging in Stockholm", Minerva, 56(2), pp. 183-207.

Burgos, A. y R. Páez (2012), "Trabas y obstáculos en la gestión local para la atención de la problemática ambiental en municipios michoacanos", Memorias de la I Bienal de Territorios en Movimiento, formato digital (CD), 18 pp.

Cabrero-Mendoza, E. (2004), "Capacidades institucionales en gobiernos subnacionales de México: ¿Un obstáculo para la descentralización fiscal?”, Gestión y Política Pública, XIII(3), pp. 753-784.

Callejo-Gallego, J. (2002), “Observación, entrevista y grupo de discusión: El silencio de tres prácticas de investigación”, Revista Española de Salud Pública, 76, pp. 409-422.

Cash, D.W., W.C. Clark, F. Alcock, N. Dickson, N. Eckley y J. Jager (2002), "Salience, Credibility, Legitimacy and Boundaries: Linking Research, Assessment and Decision Making", documento de trabajo 02-046, Universidad de Harvard-Escuela de Gobierno John F. Kennedy.

Cash, D.W., W.C. Clark, F. Alcock, N. Dickson, N. Eckley, D.H. Guston, J. Jäger y R. Mitchell (2003), "Knowledge Systems for Sustainable Development", Proceedings of the National Academy of Sciences, 100, pp. 8086-8091.

Cedemun (Centro Estatal para el Desarrollo Municipal) (2013), "Estadística de las verificaciones del periodo 2013", presentación de resultados en reunión de Cedemun Michoacán, 25 de noviembre. 
Coenen, F. (2009), “Local Agenda 21: Meaningful and Effective 'Participation?”, en F. Coenen (ed.), Public Participation and Better Environmental Decisions, Nueva York, Springer, pp. 165-182.

Conapo (Consejo Nacional de Población) (2018), Datos abiertos del Índice de Marginación, disponible en: http://www.conapo.gob.mx/es/CONAPO/Datos_Abiertos_del_Indice_de_Marginacion [fecha de consulta: 7 de agosto de 2018].

Engels, A. (2005), "The Science-policy Interface”, Integrated Assessment, 5(1), pp. 7-26.

Etzkowitz, H. y L. Leydesdorff (2000), "The Dynamics of Innovation: From National Systems and 'Mode 2' to a Triple Helix of University-Industry-Government Relations", Research Policy, 29(2), pp. 109-123.

Fam, D., L. Neuhauser y P. Gibbs (2018), Transdisciplinary Theory, Practice and Education, Cham, Springer.

Fenton, P. y S. Gustafsson (2017), "Moving from High-level Words to Local Action-Governance for Urban Sustainability in Municipalities", Current Opinion in Environmental Sustainability, 26-27, pp. 129-133.

Funtowics, S. y J. Ravetz (1993), "Science for the Post-normal Age”, Futures, 25(7), pp. 739-755.

García-Añón, M. (2004), “La Agenda 21 Local: Proceso y evaluación”, Revista Galega de Economía, 13(1-2), pp. 1-21.

Gluckman, P. (2016), “The Science-policy Interface”, Science, 353(6303), 969, Dor: 10.1126/ science.aai8837.

Heink, U., E. Marquard, K. Heubach, K. Jax, C. Kugel, C. Nesshöver, R.K. Neumann, A. Paulsch, S. Tilch, J. Timaeus, M. Vandewalle (2015), “Conceptualizing Credibility, Relevance and Legitimacy for Evaluating the Effectiveness of Science-policy Interfaces: Challenges and Opportunities", Science and Public Policy, 42(5), pp. 676-689.

Howarth, C. y J. Painter (2016), "Exploring the Science-policy Interface on Climate Change: The Role of the IPCC in Informing Local Decision-making in the UK", Palgrave Communications, 2, 16058, DoI: https://doi.org/10.1057/palcomms.2016.58.

Huitema, D. y E. Turnhout (2009), "Working at the Science-policy Interface: A Discursive Analysis of Boundary Work at the Netherlands Environmental Assessment Agency", Environmental Politics, 18(4), pp. 576-594.

Inafed (Instituto Nacional para el Federalismo y el Desarrollo Municipal) (2004), Programa Agenda desde lo Local, Ciudad de México, Secretaría de Gobernación-Instituto Nacional para el Federalismo y el Desarrollo Municipal.

Inafed (Instituto Nacional para el Federalismo y el Desarrollo Municipal) (2012), Programa Agenda desde lo Local, 7a. ed., Ciudad de México, Secretaría de GobernaciónInstituto Nacional para el Federalismo y el Desarrollo Municipal. 
Inafed (Instituto Nacional para el Federalismo y el Desarrollo Municipal) (2014), Programa Agenda para el Desarrollo Municipal, Ciudad de México, Secretaría de GobernaciónInstituto Nacional para el Federalismo y el Desarrollo Municipal.

INEGI (Instituto Nacional de Estadística y Geografía) (2016), Anuario estadístico y geográfico por entidad federativa, Ciudad de México, INEgi, $742 \mathrm{pp}$.

Jones, S.A., B. Fischhoff y D. Lach (1999), "Evaluating the Science-policy Interface for Climate Change Research", Climatic Change, 43(3), pp. 581-599.

Kawulich, B. (2005), "Participant Observation as a Data Collection Method", Forum: Qualitative Social Research, 6(2), Dor: http://dx.doi.org/10.17169/fqs-6.2.466.

Kitchen, T., D. Whitney y S. Littlewood (1997), "Local Authority/Academic Collaboration and Local Agenda 21 Policy Processes", Journal of Environmental Planning and Management, 40(5), pp. 645-660.

Klein, J.T., W. Grossenbacher-Mansuy, R. Häberli, A. Bill, R.W. Scholz y M. Welti (eds.) (2001), “Transdisciplinarity: Joint Problem Solving among Science, Technology, and Society: An Effective Way for Managing Complexity”, Springer Science \& Business Media.

Koontz, T. (2019), “The Science-policy Nexus in Collaborative Governance: Use of Science in Ecosystem Recovery Planning”, Review of Policy Research, 36(6), pp. 708-735.

Lafferty, W.M. y K. Eckerberg (eds.) (1998), From the Earth Summit to Local Agenda 21, Londres y Nueva York, Earthscan.

Leydesdorff, L. y H. Etzkowitz (1998), “The Triple Helix as a Model for Innovation Studies", Science and Public Policy, 25(3), pp. 195-203.

Lundvall, B.A., B. Johnson, E. Andersen y B. Dalum (2002), "National Systems of Production, Innovation and Competence Building", Research Policy, 31, pp. 213-231.

Max-Neef, M.A. (2005), "Foundations of Transdisciplinarity”, Ecological Economics, 53(1), pp. 5-16.

Merino, M. (2005), "Los gobiernos municipales en México: El problema del diseño institucional”, en A. Aziz Nassif y J. Alonso Sánchez (coords.), Globalización, poderes y seguridad nacional, Ciudad de México, ciesas/Miguel Ángel Porrúa/H. Cámara de Diputados-LIX Legislatura, pp. 287-305.

More, S.J. (2019), "Perspectives from the Science-policy Interface in Animal Health and Welfare”, Frontiers in Veterinary Science, 6: 382, Dor: 10.3389/fvets.2019.00382.

Nesshöver, C., J. Timaeus, H. Wittmer, A. Krieg, N. Geamana, S. van den Hove, J. Young y M. Watt (2013), “ Improving the Science-Policy Interface of Biodiversity Research Projects", GAIA-Ecological Perspectives for Science and Society, 22(2), pp. 99-103.

Nicolescu, B. (2002), Manifiesto of Transdisciplinarity, Nueva York, Universidad Estatal de Nueva York. 
Nicolescu, B. (2012), "Transdisciplinarity: The Hidden Third, between the Subject and the Object", Human and Social Studies, 1(1), pp. 13-28.

Nicolescu, B. (2014), "Methodology of Transdisciplinarity", World Futures, 70(3-4), pp. 186-199.

Nursey-Bray, M. J., J. Vince, M. Scott, M. Haward, K. O’Toole, T. Smith, N. Harvey y B. Clarke (2014), "Science into Policy? Discourse, Coastal Management and Knowledge”, Environmental Science \& Policy, 38, pp. 107-119.

Ojeda-Medina, T. (2020), "El rol estratégico de los gobiernos locales y regionales en la implementación de la Agenda 2030: Experiencias desde la cooperación Sur-Sur y triangular", Oasis, 31, pp. 9-29.

Perrings, C., A. Duraiappah, A. Larigauderie y H. Mooney (2011), "The Biodiversity and Ecosystem Services Science-policy Interface”, Science, 331(6021), pp. 1139-1140.

Piña, F. (2013), "El Programa Agenda desde lo Local: Un análisis de su valor para el desarrollo municipal”, tesis de licenciatura, Universidad Nacional Autónoma de México, $236 \mathrm{pp}$.

Ramírez, L.F. y B.M. Belcher (2019), "Stakeholder Perceptions of Scientific Knowledge in Policy Processes: A Peruvian Case-study of Forestry Policy Development", Science and Public Policy, 46(4), pp. 504-517.

Rosales, M. (2012), "Descentralización en América Latina y tareas del municipalismo”, Revista Análisis Público, 1(1), pp. 103-131.

Sancassiani, W. (2005), "Local Agenda 21 in Italy: An Effective Governance Tool for Facilitating Local Communities' Participation and Promoting Capacity Building for Sustainability", Local Environment, 10(2), pp. 189-200.

Sarkki, S., J. Niemelä, R. Tinch, S. van Den Hove, A. Watt y J. Young (2014), "Balancing Credibility, Relevance and Legitimacy: A Critical Assessment of Trade-offs in Sciencepolicy Interfaces", Science and Public Policy, 41(2), pp. 194-206.

Sarkki, S., R. Tinch, J. Niemelä, U. Heink, K. Waylen, J. Timaeus, J.C. Young, A. Watt, C. Nesshöver y S. van den Hove (2015), "Adding 'Iterativity' to the Credibility, Relevance, Legitimacy: A Novel Scheme to Highlight Dynamic Aspects of Science-policy Interfaces", Environmental Science \& Policy, 54, pp. 505-512.

Sarkki, S., E. Balian, U. Heink, H. Keune, C. Nesshöver, J. Niemelä, R. Tinch, S. van den Hove, A. Watt, K.A. Waylen y J.C. Young (2019), "Managing Science-policy Interfaces for Impact: Interactions within the Environmental Governance Meshwork", Environmental Science \& Policy, Dor: https://doi.org/10.1016/j.envsci.2019.05.011.

Saviano, M., S. Barile, F. Farioli y F. Orecchini (2019), "Strengthening the Science-policyindustry Interface for Progressing toward Sustainability: A Systems Thinking View”, Sustainability Science, 14, pp. 1549-1564. 
Selman, P. y J. Parker (1997), "Citizenship, Civicness and Social capital in Local Agenda 21”, Local Environment, 2(2), pp. 171-184.

Scarano, F.R., M.C.G. Padgurschi, A.P.F. Pires, P.F.D. Castro, J.S. Farinaci, M. Bustamante, J.P. Metzger, J.P. Ometto, C.S. Seixas y C.A. Joly (2019), "Increasing Effectiveness of the Science-policy Interface in the Socioecological Arena in Brazil", Biological Conservation, 240, DoI: https://doi.org/10.1016/j.biocon.2019.108227.

Scholz, R.W. y G. Steiner (2015), "The Real Type and Ideal Type of Transdisciplinary Processes: Part I-Theoretical Foundations", Sustainability Science, 10(4), pp. 527-544.

Slack, L. (2015), "The Post-2015 Global Agenda: A Role for Local Government", Commonwealth Journal of Local Governance, 16-17, pp. 3-11.

Sosa-López, J.D. (2012), "Gobiernos locales y desarrollo territorial en México”, Frontera Norte, 24(47), pp. 171-192.

Star, S.L. y J.R. Griesemer (1989), “Institutional Ecology, Translations' and Boundary Objects: Amateurs and Professionals in Berkeley's Museum of Vertebrate Zoology, 190739", Social Studies of Science, 19(3), pp. 387-420.

Star, S. (2010), "This Is Not a Boundary Object: Reflections on the Origin of a Concept”, Science, Technology \& Human Values, 35(5), pp. 601-617.

Steger, C., S. Hirsch, C. Evers, B. Branoff, M. Petrova, M. Nielsen-Pincus, C. Wardroper y C.J. van Riper (2018), "Ecosystem Services as Boundary Objects for Transdisciplinary Collaboration", Ecological Economics, 143, pp. 153-160.

Strydom, W.F., N. Funke, S. Nienaber, K. Nortje y M. Steyn (2010), “Evidence-based Policymaking: A Review”, South African Journal of Science, 106(5-6), pp. 17-24.

Swilling, M. (2014), "Rethinking the Science-policy Interface in South Africa: Experiments in Knowledge Co-production", South African Journal of Science, 110(5-6), pp. 1-7.

Tinch, R., E. Balian, D. Carss, D.E. de Blas, N.A. Geamana, U. Heink, H. Keune, C. Nesshöver, J. Niemelä, S. Sarkki, M. Thibon, J. Timaeus, A. Vadineanu, S, van den Hove, A. Watt, K.A. Waylen, H. Wittmer y J.C. Young (2018), "Science-policy Interfaces for Biodiversity: Dynamic Learning Environments for Successful Impact", Biodiversity and Conservation, 27(7), pp. 1679-1702.

Tonn, B. (2007), "The Intergovernmental Panel on Climate Change: A Global Scale Transformative Initiative", Futures, 39(5), pp. 614-618.

Van den Hove, S. (2007), "A Rationale for Science-policy Interfaces", Futures, 39(7), pp. 807-826.

Vohland, K., M.C. Mlambo, L.D. Horta, B. Jonsson, A. Paulsch y S.I. Martinez (2011), "How to Ensure a Credible and Efficient IPBes?", Environmental Science \& Policy, 14(8), pp. 1188-1194.

Von Der Heyden, S., P. Lukey, L. Celliers, K. Prochazka y A.T. Lombard (2016), "Science 
to Policy-Reflections on the South African Reality", South African Journal of Science, 112(11-12), pp. 1-6.

White, D.D., E.A. Corley y M.S. White (2008), "Water Managers' Perceptions of the Science-policy Interface in Phoenix, Arizona: Implications for an Emerging Boundary Organization", Society and Natural Resources, 21(3), pp. 230-243.

Young, J.C., K.A. Waylen, S. Sarkki, S. Albon, I. Bainbridge, E. Balian, J. Davidson, D. Edwards, R. Fairley, C. Margerison, D. McCracken, R. Owen, C.P. Quine, C. Stewart-Roper, D. Thompson, R. Tinch, S. van den Hove y A. Watt (2014), "Improving the Science-policy Dialogue to Meet the Challenges of Biodiversity Conservation: Having Conversations Rather than Talking at One-another", Biodiversity and Conservation, 23(2), pp. 387-404. Zheng, Y., L.A. Naylor, S. Waldron y D.M. Oliver (2019), "Knowledge Management a Cross the Environment-policy Interface in China: What Knowledge is Exchanged, Why, and How is This Undertaken?", Environmental Science \& Policy, 92, pp. 66-75.

Ziccardi, A. y H. Saltalamacchia (1997), Metodología de evaluación del desempeño de los gobiernos locales en ciudades mexicanas, Ciudad de México, UNAM-IIS.

Ana L. Burgos es bióloga por la Universidad del Comahue (Argentina) y doctora en Ciencias por la UnAM. Miembro del Sistema Nacional de Investigadores (SNI) nivel I, y fuerte promotora de la circulación del conocimiento científico en la sociedad. Sus temas de interés incluyen la investigación transdisciplinaria para la gestión de cuencas hidrográficas y el desarrollo sostenible, y la innovación rural en contextos territoriales rezagados.

Gerardo Bocco es geógrafo por la UnAM y doctor en Geografía por la Universidad de Ámsterdam. Investigador titular de la UnAm desde 1995. Miembro del sNi y de la Academia Mexicana de Ciencias. Su interés académico principal se enfoca en la geografía de pequeñas localidades rurales en América Latina. 